\title{
Introduction to and Strengthening of the Atiyah-Sutcliffe Conjectures for Several Types of four points Configurations
}

\author{
Dragutin Svrtan \\ Department of Mathematics, University of Zagreb, \\ Bijenička cesta 30, 10000 Zagreb, Croatia, \\ dsvrtan@math.hr \\ Igor Urbiha \\ Department of Informatics, Polytechnic of Zagreb, University of Zagreb, \\ Konavoska 2, 10000 Zagreb, Croatia, \\ urbiha@vtszg.hr \\ AMS Subject Classifications: 74H05, 11B37, 26A18, 05A15, 11Y55, 11 Y65
}

\begin{abstract}
In 2001 Sir M. F. Atiyah formulated a conjecture C1 and later with P. Sutcliffe two stronger conjectures C2 and C3. These conjectures, inspired by physics (spin-statistics theorem of quantum mechanics), are geometrically defined for any configuration of points in the Euclidean three space. The conjecture $\mathrm{C} 1$ is proved for $n=3,4$ and for general $n$ only for some special configurations (M. F. Atiyah, M. Eastwood and P. Norbury, D.Đoković). Interestingly the conjecture C2 (and also stronger C3) is not yet proven even for arbitrary four points in a plane. So far we have verified the conjectures $\mathrm{C} 2$ and $\mathrm{C} 3$ for parallelograms, cyclic quadrilaterals and some infinite families of tetrahedra.

We have also proposed a strengthening of conjecture C3 for configurations of four points (Four Points Conjectures).

For almost collinear configurations (with all but one point on a line) we propose several new conjectures (some for symmetric functions) which imply C2 and C3. By using computations with multi-Schur functions we can do verifications up to $n=9$ of our conjectures. We can also verify stronger conjecture of Đoković which imply C2 for his nonplanar configurations with dihedral symmetry.

Finally we mention that by minimizing a geometrically defined energy, figuring in these conjectures, one gets a connection to some complicated physical theories, such as Skyrmions and Fullerenes.
\end{abstract}

DOI: https://doi.org/10.5592/CO/CCD.2020.07 


\section{Introduction on Geometric Energies}

In this Section we describe some geometric energies, introduced by Atiyah. To construct first geometric energy consider $n$ distinct ordered points, $\mathbf{x}_{i} \in \mathbb{R}^{3}$ for $i=1, \ldots, n$. For each pair $i \neq j$ define the unit vector

$$
\mathbf{v}_{i j}=\frac{\mathbf{x}_{j}-\mathbf{x}_{i}}{\left|\mathbf{x}_{j}-\mathbf{x}_{i}\right|}
$$

giving the direction of the line joining $\mathbf{x}_{i}$ to $\mathbf{x}_{j}$. Now let $t_{i j} \in \mathbb{C P}^{1}$ be the point on the Riemann sphere associated with the unit vector $\mathbf{v}_{i j}$, via the identification $\mathbb{C P}^{1} \cong S^{2}$, realized as stereographic projection. Next, set $p_{i}$ to be the polynomial in $t$ with roots $t_{i j}(j \neq i)$, that is

$$
p_{i}=\alpha_{i} \prod_{j \neq i}\left(t-t_{i j}\right)
$$

where $\alpha_{i}$ is a certain normalization coefficient. In this way we have constructed $n$ polynomials which all have degree $n-1$, and so we may write

$$
p_{i}=\sum_{j=1}^{n} m_{i j} t^{j-1} \text {. }
$$

Finally, let $M_{n}$ be the $n \times n$ matrix with entries $m_{i j}$, and let $\mathcal{D}_{n}$ be its determinant

$$
\mathcal{D}_{n}=\mathcal{D}_{n}\left(\mathbf{x}_{1}, \ldots, \mathbf{x}_{n}\right)=\operatorname{det} M_{n}
$$

This geometrical construction is relevant to the Berry-Robbins problem, which is concerned with specifying how a spin basis varies as $n$ point particles move in space, and supplies a solution provided it can be shown that $\mathcal{D}_{n}$ is always non-zero. For $n=2,3,4$ it can be proved that $\mathcal{D}_{n} \neq 0$ (Atiyah $n=3$, Eastwood and Norbury $n=4$ ) and numerical computations suggest that $\left|\mathcal{D}_{n}\right| \geq 1$ for all $n$, with the minimal value $\left|\mathcal{D}_{n}\right|=1$ being attained by $n$ collinear points.

The geometric energy is the $n$-point energy defined by

$$
E_{n}=-\log \left|\mathcal{D}_{n}\right|
$$

so minimal energy configurations maximize the modulus of the determinant.

This energy is geometrical in the sense that it only depends on the directions of the lines joining the points, so it is translation, rotation and scale invariant. Remarkably, the minimal energy configurations, studied numerically for all $n \leq$ 32 , are essentially the same as those for the Thomson problem.

\section{Eastwood-Norbury formulas for Atiyah de- terminants}

In this section we first recall Eastwood-Norbury formula for Atiyah determinant for three or four points in Euclidean three-space. In the case $n=3$ the (non 
normalized) Atiyah determinant reads as

$$
D_{3}=d_{3}\left(r_{12}, r_{13}, r_{23}\right)+8 r_{12} r_{13} r_{23}
$$

where

$$
d_{3}(a, b, c)=(a+b-c)(b+c-a)(c+a-b)
$$

and $r_{i j}(1 \leq i<j \leq 3)$ is the distance between the $i^{\text {th }}$ and $j^{\text {th }}$ point.

The normalized Atiyah determinant for 3 points is

$$
\mathcal{D}_{3}=\frac{D_{3}}{8 r_{12} r_{13} r_{23}}
$$

and it is evident that $\left|\mathcal{D}_{3}\right|=\mathcal{D}_{3} \geq 1$.

In the case $n=4$ the (non normalized) Atiyah determinant $D_{4}$ has real part given by a polynomial (with 248 terms) as follows:

$$
\Re\left(D_{4}\right)=64 r_{12} r_{13} r_{23} r_{14} r_{24} r_{34}-4 d_{3}\left(r_{12} r_{34}, r_{13} r_{24}, r_{14} r_{23}\right)+A_{4}+288 V^{2}
$$

where

$$
A_{4}=\sum_{l=1}^{4}\left(\sum_{(l \neq) i=1}^{4} r_{l i}\left(\left(r_{l j}+r_{l k}\right)^{2}-r_{j k}^{2}\right)\right) d_{3}\left(r_{i j}, r_{i k}, r_{j k}\right)
$$

(here $\{j, k\}=\{1,2,3,4\} \backslash\{l, i\})$ and $V$ denotes the volume of the tetrahedron with vertices our four points:

$$
\begin{aligned}
144 V^{2}= & r_{12}^{2} r_{34}^{2}\left(r_{13}^{2}+r_{14}^{2}+r_{23}^{2}+r_{24}^{2}-r_{12}^{2}-r_{34}^{2}\right)+\text { two similar terms } \\
& -\left(r_{12}^{2} r_{13}^{2} r_{23}^{2}+\text { three similar terms }\right)
\end{aligned}
$$

We now state two formulas which will be used later:

1. Alternative form of $A_{4}$ :

$$
\begin{gathered}
A_{4}=\sum_{l=1}^{4}\left(\left(d_{3}\left(r_{i l}, r_{j l}, r_{k l}\right)+8 r_{i l} r_{j l} r_{k l}+r_{i l}\left(r_{i l}^{2}-r_{j k}^{2}\right)+\right.\right. \\
\left.r_{j l}\left(r_{j l}^{2}-r_{i k}^{2}\right)+r_{k l}\left(r_{k l}^{2}-r_{i j}^{2}\right)\right) d_{3}\left(r_{i j}, r_{i k}, r_{j k}\right),
\end{gathered}
$$

where for each $l$ we write $\{1,2,3,4\} \backslash\{l\}=\{i<j<k\}$.

2. The sum of the second and the fourth term of (2.5) can be rewritten as

$$
\begin{aligned}
144 V^{2}- & 2 d_{3}\left(r_{12} r_{34}, r_{13} r_{24}, r_{14} r_{23}\right)= \\
= & \left(r_{12}-r_{34}\right)^{2}\left(r_{13}^{2} r_{24}^{2}+r_{14}^{2} r_{23}^{2}-r_{12}^{2} r_{34}^{2}\right)+\text { two such terms }+ \\
& +4 r_{12} r_{13} r_{23} r_{14} r_{24} r_{34}- \\
& -r_{12}^{2} r_{13}^{2} r_{23}^{2}-r_{12}^{2} r_{14}^{2} r_{24}^{2}-r_{13}^{2} r_{14}^{2} r_{34}^{2}-r_{23}^{2} r_{24}^{2} r_{34}^{2} .
\end{aligned}
$$


It is well known that this quantity is always nonpositive.

The imaginary part $\Im\left(D_{4}\right)$ ) of Atiyah determinant can be written as a product of $144 V^{2}$ with a polynomial (with integer coefficients) having 369 terms.

The normalized Atiyah determinant for 4 points is

$$
\mathcal{D}_{4}=\frac{D_{4}}{2^{\left(\begin{array}{l}
4 \\
2
\end{array}\right)} \prod_{1 \leq i<j \leq 4} r_{i j}} .
$$

The original Atiyah conjecture in our cases is equivalent to nonvanishing of the determinants $D_{3}$ and $D_{4}$.

A stronger conjecture of Atiyah and Sutcliffe ([4],Conjecture 2) states in our cases that $\left|D_{3}\right| \geq 8 r_{12} r_{13} r_{23}\left(\Leftrightarrow\left|\mathcal{D}_{3}\right| \geq 1\right)$ and $\left|D_{4}\right| \geq 64 r_{12} r_{13} r_{23} r_{14} r_{24} r_{34}$ $\left(\Leftrightarrow\left|\mathcal{D}_{4}\right| \geq 1\right)$.

From the formula (2.5) above, with the help of the simple inequality $d_{3}(a, b, c)$ $\leq a b c$ (for $a, b, c \geq 0$ ), Eastwood and Norbury got "almost" the proof of the stronger conjecture by exhibiting the inequality

$$
\Re\left(D_{4}\right) \geq 60 r_{12} r_{13} r_{23} r_{14} r_{24} r_{34} .
$$

To remove the word "almost" seems to be not so easy (at present not yet done even for planar configuration of four points).

A third conjecture (stronger than the second) of Atiyah and Sutcliffe ([4], Conjecture 3) can be expressed, in the four point case, in terms of polynomials in the edge lengths as

$$
\left|D_{4}\right|^{2} \geq \prod_{\{i<j<k\} \subset\{1,2,3,4\}}\left(d_{3}\left(r_{i j}, r_{i k}, r_{j k}\right)+8 r_{i j} r_{i k} r_{j k}\right)
$$

where the product runs over the four faces of the tetrahedron.

(cf. ftp://ftp.maths.adelaide.edu.au/pure/meastwood/atiyah.ps)

In the first part of this paper we study some infinite families of quadrilaterals and tetrahedra and verify both Atiyah and Sutcliffe conjectures for several such infinite families. In this version of the paper we propose a somewhat stronger conjecture than (2.9) which reads as follows:

Conjecture 2.1 (Four Points Conjectures)

$$
\begin{aligned}
& \Re\left(D_{4}\right)-\left(4+\frac{3}{4}\right) \cdot 288 V^{2} \geq \\
& \geq 64 \prod_{1 \leq i, j \leq 4} r_{i j}+\sum_{\{i<j<k\} \subset\{1,2,3,4\}}\left(4+\frac{1}{4} \delta\right) r_{i l} r_{j l} r_{k l} d_{3}\left(r_{i j}, r_{i k}, r_{j k}\right)
\end{aligned}
$$

where

$$
\delta=\left\{\begin{array}{cl}
\frac{d_{3}\left(r_{i j}, r_{i k}, r_{j k}\right)}{r_{i j} r_{i k} r_{j k}} & , \text { weak version } \\
1, & \text { strong version }
\end{array}\right.
$$


Proposition 2.2 Any of the Four Points Conjectures (2.10) imply conjecture (2.9).

\section{Proof .}

By using the inequality $1 \geq d_{3}(a, b, c) /(a b c),(a, b, c>0)$ (see Appendix 2, Proposition 2.13) we see that the strong version implies the weak version of conjecture. We then rewrite the rhs of the weak version of (2.10) as follows:

$$
\prod_{1 \leq i, j \leq 4} r_{i j}\left(\frac{1}{4} \sum_{l=1}^{4}\left(\frac{d_{3}\left(r_{i j}, r_{i k}, r_{j k}\right)}{r_{i j} r_{i k} r_{j k}}+8\right)^{2}\right)
$$

Finally, by the quadratic-geometric $(\mathrm{QG})$ inequality we obtain

$$
\geq \prod_{1 \leq i, j \leq 4} r_{i j}\left(\prod_{l=1}^{4}\left(\frac{d_{3}\left(r_{i j}, r_{i k}, r_{j k}\right)}{r_{i j} r_{i k} r_{j k}}+8\right)\right)^{\frac{2}{4}}=\left(\prod_{l=1}^{4}\left(d_{3}\left(r_{i j}, r_{i k}, r_{j k}\right)+8 r_{i j} r_{i k} r_{j k}\right)\right)^{\frac{1}{2}}
$$

Thus we obtain:

$$
\left|D_{4}\right|^{2} \geq\left|\Re\left(D_{4}\right)\right|^{2} \geq\left|\Re\left(D_{4}\right)-\left(4+\frac{3}{4}\right) \cdot 288 V^{2}\right|^{2} \geq \prod_{l=1}^{4}\left(d_{3}\left(r_{i j} r_{i k} r_{j k}\right)+8 r_{i j} r_{i k} r_{j k}\right)
$$

i.e. the inequality (2.9).

Remark 2.3 In terms of trigonometry (see subsection "Atiyah determinant for triangles and quadrilaterals via trigonometry" on page 114), the weak Four Points Conjecture can be written simply as

$$
\Re\left(D_{4}\right)-\left(4+\frac{3}{4}\right) \cdot 288 V^{2} \geq\left(\prod_{1 \leq i, j \leq 4} r_{i j}\right)\left(4 \sum_{l=1}^{4} c_{l}^{2}\right)
$$

where

$$
c_{l}:=\cos ^{2} \frac{X^{(l)}}{2}+\cos ^{2} \frac{Y^{(l)}}{2}+\cos ^{2} \frac{Z^{(l)}}{2}, \quad l=1,2,3,4 .
$$

and $X^{(l)}, Y^{(l)}, Z^{(l)}$ are the angles of the triangle opposite to the vertex $l$.

\subsection{Atiyah-Sutcliffe conjecture for (vertically) upright tetra- hedra (or pyramids)}

We call a tetrahedron upright if some of its vertices (say 4) is equidistant from all the remaining vertices $(1,2$ and 3 , which we can think as lying in a horizontal plane.) 


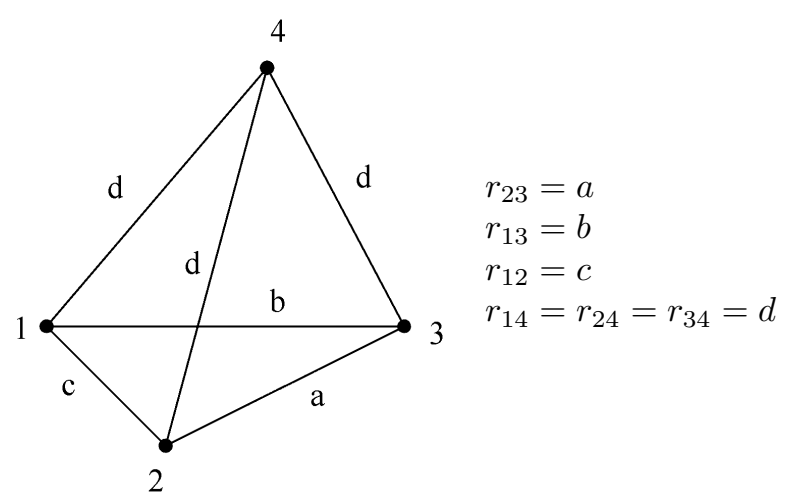

Note that then $d \geq R=$ the circumradius of the base triangle 123 , then by Heron's formula we have: $R=a b c / \sqrt{(a+b+c) d_{3}(a, b, c)}$.

Here, as before, $d_{3}(a, b, c)=(a+b-c)(a-b+c)(-a+b+c),(a, b, c>0)$.

The left hand side of the strong Four Points Conjecture 2.10 (but without $\frac{3}{4}$ term!) can be evaluated as follows, by using Eastwood-Norbury formula (2.5)

$$
\begin{aligned}
\text { LHS } & =\Re\left(D_{4}\right)-4 \cdot 288 V^{2}= \\
& =64 \prod_{1 \leq i<j \leq 4} r_{i j}-4 d_{3}\left(r_{12} r_{34}, r_{13} r_{24}, r_{14} r_{23}\right)+A_{4}-3 \cdot 288 V^{2}
\end{aligned}
$$

where

$$
\begin{aligned}
&-4 d_{3}\left(r_{12} r_{34}, r_{13} r_{24}, r_{14} r_{23}\right)=-4 d_{3}(a, b, c) d^{3} \\
& A_{4}= \sum_{l=1}^{4}\left(\sum_{(l \neq) i=1}^{4} r_{l i}\left(\left(r_{l j}+r_{l k}\right)^{2}-r_{j k}^{2}\right) d_{3}\left(r_{i j}, r_{i k}, r_{j k}\right)\right)= \\
&= \sum_{c y c(a, b, c)}\left[c\left((b+d)^{2}-d^{2}\right)+b\left((c+d)^{2}-d^{2}\right)+d\left((b+c)^{2}-a^{2}\right)\right] d_{3}(a, d, d)+ \\
& \quad \quad\left[d\left((d+d)^{2}-a^{2}\right)+d\left((d+d)^{2}-b^{2}\right)+d\left((d+d)^{2}-c^{2}\right)\right] d_{3}(a, b, c)= \\
&=\left.\quad\left[4 b c d+\left((b+c)^{2}-a^{2}\right) d+b^{2} c+b c^{2}\right]\left(2 a^{2} d-a^{3}\right)\right]+\cdots+ \\
& \quad+\left[12 d^{3}-\left(a^{2}+b^{2}+c^{2}\right) d\right] d_{3}(a, b, c) \\
&-3 \cdot 288 V^{2}= \quad(b y) \\
&=-6\left[\left(b^{2}+c^{2}-a^{2}\right) a^{2} d^{2}+\left(c^{2}+a^{2}-b^{2}\right) b^{2} d^{2}+\left(a^{2}+b^{2}-c^{2}\right) c^{2} d^{2}-a^{2} b^{2} c^{2}\right](b y 2.6) \\
&=-6\left[\left(2 a^{2} b^{2}+2 a^{2} c^{2}+2 b^{2} c^{2}\right) d^{2}-a^{2} b^{2} c^{2}\right]-a^{4}-b^{4}-c^{4} \\
&=-6\left[(a+b+c) d_{3}(a, b, c) d^{2}-a^{2} b^{2} c^{2}\right]
\end{aligned}
$$


Similarly the right hand side of the Conjecture 2.10

$$
\begin{aligned}
R H S= & 64 \prod_{1 \leq i<j \leq 4} r_{i j}+\sum_{l=1}^{4}\left(4+\frac{1}{4}\right) r_{i l} r_{j l} r_{k l} d_{3}\left(r_{i j}, r_{i k}, r_{j k}\right) \\
= & 64 a b c d^{3}+\left(4+\frac{1}{4}\right) b c d\left(2 a^{2} d-a^{3}\right)+\text { two such terms }+ \\
& +\left(4+\frac{1}{4}\right) d^{3} d_{3}(a, b, c)
\end{aligned}
$$

Now we can rewrite the difference

$$
L H S-R H S=I+I I
$$

where

$$
I=\sum_{c y c}\left(b^{2} c+b c^{2}\right)\left(2 a^{2} d-a^{3}\right)-\left(a^{2}+b^{2}+c^{2}\right) d_{3}(a, b, c) d+6 a^{2} b^{2} c^{2}-24 \frac{a^{2} b^{2} c^{2}}{a+b+c} d
$$

and

$$
\begin{aligned}
I I= & \left(4-\frac{1}{4}\right) d_{3}(a, b, c) d^{3}+\sum_{c y c}\left((b+c)^{2}-a^{2}\right) d\left(2 a^{2} d-a^{3}\right)- \\
& -6 \sum_{c y c}\left(b^{2}+c^{2}-a^{2}\right) a^{2} d^{2}-\frac{1}{4} \sum_{c y c} b c d\left(2 a^{2} d-a^{3}\right)+24 \frac{a^{2} b^{2} c^{2}}{a+b+c} d
\end{aligned}
$$

Then we can further simplify

$$
\begin{aligned}
I= & {\left[4 a b c(a b+a c+b c)-\left(a^{2}+b^{2}+c^{2}\right) d_{3}(a, b, c)-\frac{24 a^{2} b^{2} c^{2}}{a+b+c}\right] d+} \\
& +6 a^{2} b^{2} c^{2}-\sum_{\text {sym }} a^{3} b^{2} c
\end{aligned}
$$

and

$$
\begin{aligned}
I I= & d\left[\frac{15}{4} d_{3}(a, b, c) d^{2}+(a+b+c)\left(\frac{7}{2} a b c-4 d_{3}(a, b, c)\right) d+24 \frac{a^{2} b^{2} c^{2}}{a+b+c}+\right. \\
& \left.+\frac{1}{4} a b c\left(a^{2}+b^{2}+c^{2}\right)-(a+b+c)\left(\sum_{\text {sym }} a^{3} b-a^{4}-b^{4}-c^{4}\right)\right]
\end{aligned}
$$

Lemma 2.4 We have the following strengthening of the basic inequality for our function $d_{3}(a, b, c)=(a+b-c)(a-b+c)(-a+b+c)$ :

$$
d_{3}(a, b, c) \leq \frac{9 a^{2} b^{2} c^{2}}{(a+b+c)\left(a^{2}+b^{2}+c^{2}\right)} \quad\left(\leq \frac{27 a^{2} b^{2} c^{2}}{(a+b+c)^{3}} \leq a b c\right)
$$




\section{Proof .}

We have

$$
\begin{aligned}
& 9 a^{2} b^{2} c^{2}-\left(a^{2}+b^{2}+c^{2}\right)(a+b+c) d_{3}(a, b, c)= \\
& =9 a^{2} b^{2} c^{2}-\left(a^{2}+b^{2}+c^{2}\right)\left(2 a^{2} b^{2}+2 a^{2} c^{2}+2 b^{2} c^{2}-a^{4}+b^{4}+c^{4}\right)= \\
& =3 a^{2} b^{2} c^{2}-a^{4} b^{2}-a^{2} b^{4}-a^{4} c^{2}-a^{2} c^{4}-b^{4} c^{2}-b^{2} c^{4}+a^{6}+b^{6}+c^{6}= \\
& \left(a^{2}-b^{2}\right)\left[a^{2}\left(a^{2}-c^{2}\right)-b^{2}\left(b^{2}-c^{2}\right)\right]+c^{2}\left(a^{2}-c^{2}\right)\left(b^{2}-c^{2}\right) \geq 0 \\
& (\text { if we assume } a \geq b \geq c \geq 0)
\end{aligned}
$$

(a special instance of a Schur inequality)

(Note that this result follows from the formula $O G^{2}=R^{2}-\left(a^{2}+b^{2}+c^{2}\right) / 9$ for the distance of the circumcenter and the centroid of a triangle.)

Now we have

Lemma 2.5 The quantity $I$ is increasing w.r.t. $d$ and it is positive for $d \geq R$.

Proof .

We prove that the coefficient of $d$ in $I$ is positive by using that $(a b+a c+b c)(a+$ $b+c) \geq 9 a b c$ and Lemma 2.4 .

The proof of positivity of $I$ reduces to the positivity of the following quantity:

$$
\begin{aligned}
& \left\{\left[4 a b c(a b+a c+b c)-\left(a^{2}+b^{2}+c^{2}\right) d_{3}(a, b, c)\right](a+b+c)-24 a^{2} b^{2} c^{2}\right\}^{2}- \\
& -(a+b+c)^{3}\left(a^{2} b+a b^{2}+a^{2} c+a c^{2}+b^{2} c+b c^{2}-6 a b c\right)^{2} d_{3}(a, b, c)
\end{aligned}
$$

which by substituting $a=b+h$ and $b=c+k$ and then expanding has all coefficients positive (and ranging from 1 to 32151 ).

Lemma 2.6 The quantity II is increasing w.r.t. $d$ and it is positive for $d \geq R$.

\section{Proof .}

Let $I I=d \cdot I I I$. Then

$$
\frac{\partial I I I}{\partial d}=\left(\frac{15}{2} d_{3}(a, b, c) d-\frac{a+b+c}{2} d_{3}(a, b, c)\right)+\frac{7(a+b+c)}{2}\left(a b c-d_{3}(a, b, c)\right)
$$

The second term is positive by Proposition 2.13. For the first term we have:

$$
\begin{aligned}
& \frac{15}{2} d_{3}(a, b, c) d-\frac{a+b+c}{2} d_{3}(a, b, c) \geq \frac{15}{2} d_{3}(a, b, c) R-\frac{a+b+c}{2} d_{3}(a, b, c) \geq \\
& \left(\frac{15 a b c}{(a+b+c)^{3 / 2}}-\sqrt{d_{3}(a, b, c)}\right) \frac{a+b+c}{2} \sqrt{d_{3}(a, b, c)} \geq 0
\end{aligned}
$$

by Lemma (2.4).

The proof of positivity of $I I$ reduces to the positivity of the following quantity:

$$
\begin{aligned}
& \frac{15}{4} d_{3}(a, b, c) R^{2}+(a+b+c)\left(\frac{7}{2} a b c-4 d_{3}(a, b, c)\right) R+24 \frac{a^{2} b^{2} c^{2}}{a+b+c}+ \\
& +\frac{1}{4} a b c\left(a^{2}+b^{2}+c^{2}\right)-(a+b+c)\left(\sum_{s y m} a^{3} b-a^{4}-b^{4}-c^{4}\right)
\end{aligned}
$$

which can be nicely visualized by Maple using tangential coordinates $(a=v+w$, $b=u+w, c=u+v)$. 


\subsection{Atiyah-Sutcliffe conjectures for edge-tangential tetra- hedra}

By edge-tangential tetrahedron we shall mean any tetrahedron for which there exists a sphere touching all its edges (i.e. its 1-skeleton has an inscribed sphere.) For each $i$ from 1 to 4 we denote by $t_{i}$ the length of the segment (lying on the tangent line) with one endpoint the vertex and the other the point of contact of the tangent line with a sphere.

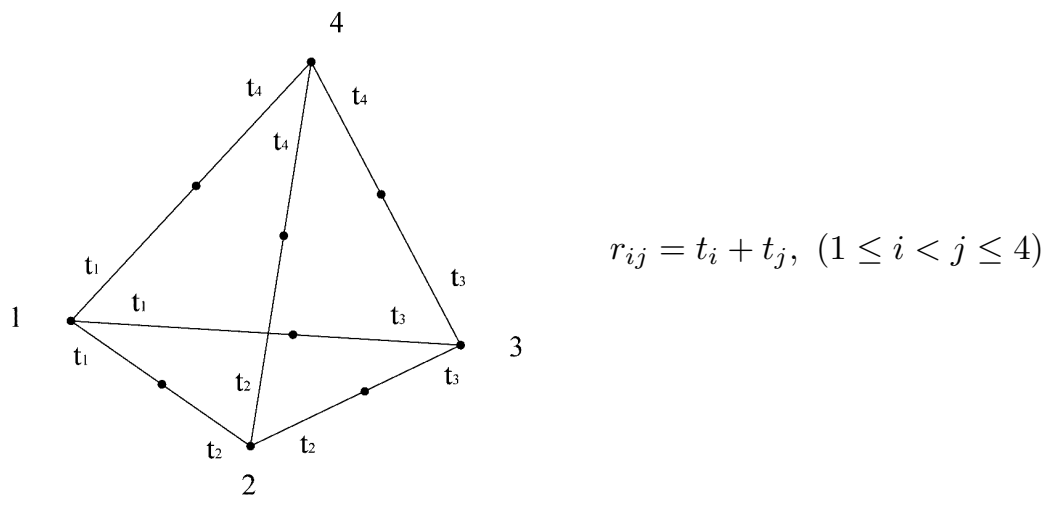

Now we shall compute all the ingredients appearing in the Eastwood-Norbury formula for $D_{4}$ in terms of elementary symmetric functions of the (tangential) variables $t_{1}, t_{2}, t_{3}, t_{4}\left(\right.$ recall $e_{1}=t_{1}+t_{2}+t_{3}+t_{4}, e_{2}=t_{1} t_{2}+t_{1} t_{3}+t_{1} t_{4}+t_{2} t_{3}+$ $\left.t_{2} t_{4}+t_{3} t_{4}, e_{3}=t_{1} t_{2} t_{3}+t_{1} t_{2} t_{4}+t_{1} t_{3} t_{4}+t_{2} t_{3} t_{4}, e_{4}=t_{1} t_{2} t_{3} t_{4}\right)$.

$$
\begin{aligned}
64 r_{12} r_{13} r_{23} r_{14} r_{24} r_{34} & =64 \prod_{1 \leq i<j \leq 4}\left(t_{i}+t_{j}\right)=64 s_{3,2,1}= \\
& =64\left|\begin{array}{ccc}
e_{3} & e_{4} & 0 \\
e_{1} & e_{2} & e_{3} \\
0 & 1 & e_{1}
\end{array}\right|=64 e_{3} e_{2} e_{1}-64 e_{4} e_{1}^{2}-64 e_{3}^{2}
\end{aligned}
$$

Here we have used Jacobi-Trudi formula for the triangular Schur function $s_{3,2,1}$ (see [10], (3.5)). Furthermore we have

$$
\begin{aligned}
-4 d_{3}\left(r_{12} r_{34}, r_{13} r_{24}, r_{14} r_{23}\right) & =128 e_{4} e_{2}-32 e_{4} e_{1}^{2}-32 e_{3}^{2} \\
288 V^{2} & =128 e_{4} e_{2}-32 e_{3}^{2}
\end{aligned}
$$

In order to compute $A_{4}$ we first compute, for fixed $l$ the following quantities

$$
\begin{aligned}
d_{3}\left(r_{i j}, r_{i k}, r_{j k}\right) & =8 t_{i} t_{j} t_{k} \\
\sum_{(l \neq) i=1}^{4} r_{l i}\left(\left(r_{l j}+r_{l k}\right)^{2}-r_{j k}^{2}\right) & =4\left(3 t_{l}\left(t_{1}+t_{2}+t_{3}+t_{4}\right)+2\left(t_{i} t_{j}+t_{i} t_{k}+t_{j} t_{k}\right)\right) t_{l} .
\end{aligned}
$$

Thus we get:

$$
A_{4}=32\left(3 e_{1}^{2}+4 e_{2}\right) e_{4}=96 e_{4} e_{1}^{2}+128 e_{4} e_{2} .
$$


Now we adjust terms in $D_{4}$, in order to get shorter expression, as follows

$$
\begin{aligned}
D_{4}= & \left(64 r_{12} r_{13} r_{23} r_{14} r_{24} r_{34}-2 \cdot 288 V^{2}\right)+ \\
& +\left(-4 d_{3}\left(r_{12} r_{34}, r_{13} r_{24}, r_{14} r_{23}\right)-288 V^{2}\right)+A_{4}+4 \cdot 288 V^{2} \\
= & \left(64 e_{3} e_{2} e_{1}-64 e_{4} e_{1}^{2}-256 e_{4} e_{2}\right)+\left(-32 e_{4} e_{1}^{2}\right)+ \\
& +\left(96 e_{4} e_{1}^{2}+128 e_{4} e_{2}\right)+4 \cdot 288 V^{2} \\
= & 64 e_{3} e_{2} e_{1}-128 e_{4} e_{2}+1152 V^{2} \\
= & 64 e_{2}\left(e_{3} e_{1}-2 e_{4}\right)+1152 V^{2} \\
= & 64 e_{2}\left(2 e_{4}+m_{211}\right)+1152 V^{2},
\end{aligned}
$$

where $m_{211}=t_{1}^{2} t_{2} t_{3}+\cdots$ denotes the monomial symmetric function associated to the partition $(2,1,1)$.

In order to verify the third conjecture of Atiyah and Sutcliffe

$$
\left|D_{4}\right|^{2} \geq \prod_{\{i<j<k\} \subset\{1,2,3,4\}}\left(d_{3}\left(r_{i j}, r_{i k}, r_{j k}\right)+8 r_{i j} r_{i k} r_{j k}\right)
$$

we note first that

$$
\begin{aligned}
d_{3}\left(r_{i j}, r_{i k}, r_{j k}\right)+8 r_{i j} r_{i k} r_{j k} & =\left(8 t_{i} t_{j} t_{k}+8\left(t_{i}+t_{j}\right)\left(t_{i}+t_{k}\right)\left(t_{j}+t_{k}\right)\right) \\
& =8\left(t_{i}+t_{j}+t_{k}\right)\left(t_{i} t_{j}+t_{i} t_{k}+t_{j} t_{k}\right)
\end{aligned}
$$

and state the following:

Lemma 2.7 For any nonnegative real numbers $t_{1}, t_{2}, t_{3}, t_{4} \geq 0$ the following inequality

$$
\begin{aligned}
& \left(t_{1} t_{2}+t_{1} t_{3}+t_{1} t_{4}+t_{2} t_{3}+t_{2} t_{4}+t_{3} t_{4}\right)^{2}\left(2 t_{1} t_{2} t_{3} t_{4}+m_{211}\left(t_{1}, t_{2}, t_{3}, t_{4}\right)\right)^{2} \geq \\
& \geq \prod_{\{i<j<k\} \subset\{1,2,3,4\}}\left(t_{i}+t_{j}+t_{k}\right)\left(t_{i} t_{j}+t_{i} t_{k}+t_{j} t_{k}\right)
\end{aligned}
$$

holds true.

\section{Proof of Lemma 2.7.}

The difference between the left hand side and the right hand side of the above inequality (2.11), written in terms of monomial symmetric functions is equal to

$$
\begin{aligned}
\text { LHS }- \text { RHS }= & m_{6321}+3 m_{6222}+m_{543}+2 m_{5421}+7 m_{5322}+5 m_{5331}+ \\
& +3 m_{444}+7 m_{4431}+8 m_{4422}+8 m_{4332}+3 m_{3333} \geq 0
\end{aligned}
$$

Remark 2.8 One may think that the inequality in Lemma 2.7 can be obtained as a product of two simpler inequalities. This is not the case, because the following inequalities hold true:

$$
\begin{aligned}
\left(t_{1} t_{2}+t_{1} t_{3}+t_{1} t_{4}+t_{2} t_{3}+t_{2} t_{4}+t_{3} t_{4}\right)^{2} & \leq \prod_{\{i<j<k\} \subset\{1,2,3,4\}}\left(t_{i}+t_{j}+t_{k}\right) \\
\left(2 t_{1} t_{2} t_{3} t_{4}+m_{211}\left(t_{1}, t_{2}, t_{3}, t_{4}\right)\right)^{2} & \geq \prod_{\{i<j<k\} \subset\{1,2,3,4\}}\left(t_{i} t_{j}+t_{i} t_{k}+t_{j} t_{k}\right)
\end{aligned}
$$


Now we continue with verification of the third conjecture of Atiyah and Sutcliffe for edge tangential tetrahedron:

$$
\begin{aligned}
\left|D_{4}\right|^{2} & \geq\left(D_{4}\right)^{2} \geq\left[64 e_{2}\left(2 e_{4}+m_{211}\right)\right]^{2} \\
& \geq 8^{4} \prod_{\{i<j<k\} \subset\{1,2,3,4\}}\left(t_{i}+t_{j}+t_{k}\right)\left(t_{i} t_{j}+t_{i} t_{k}+t_{j} t_{k}\right) \quad \text { (by Lemma 2.7) } \\
& =\prod_{\{i<j<k\} \subset\{1,2,3,4\}}\left(d_{3}\left(r_{i j}, r_{i k}, r_{j k}\right)+8 r_{i j} r_{i k} r_{j k}\right)
\end{aligned}
$$

so the strongest Atiyah-Sutcliffe conjecture is verified for edge-tangential tetrahedra.

\subsection{Verification of the strong Four Points Conjecture for edge-tangential tetrahedra}

The strong Four Points Conjecture 2.10 for edge tangential tetrahedra is equivalent to positivity of the following quantity:

$$
\begin{aligned}
& \Re\left(D_{4}\right)-64 \prod r_{i j}-\left(4+\frac{3}{4}\right) 288 V^{2}-\sum_{l=1}^{4}\left(4+\frac{1}{4}\right) r_{i l} r_{j l} r_{k l} d_{3}\left(r_{i j}, r_{i k}, r_{j k}\right) \\
&=\left(-4 d_{3}\left(r_{12} r_{34}, r_{13} r_{24}, r_{14} r_{23}\right)+A_{4}\right)+288 V^{2}-\left(4+\frac{3}{4}\right) 288 V^{2} \\
&-\sum_{l=1}^{4}\left(4+\frac{1}{4}\right) r_{i l} r_{j l} r_{k l} d_{3}\left(r_{i j}, r_{i k}, r_{j k}\right) \\
&=\left(-32 m_{3111}-32 m_{222}+96 m_{3111}+320 m_{2211}\right)-240 m_{2211}+120 m_{222} \\
&-\left(34 m_{3111}+136 m_{2211}+34 m_{222}\right) \\
&= 30 m_{3111}+54 m_{222}-56 m_{2211}
\end{aligned}
$$

In terms of augmented monomial symmetric functions

$$
\widetilde{m}_{\lambda}\left(t_{1}, t_{2}, t_{3}, t_{4}\right)=\sum_{\sigma \in S_{4}} t^{\sigma . \lambda}
$$

the last quantity is equal to

$$
=5 \widetilde{m}_{3111}+9 \widetilde{m}_{222}-14 \widetilde{m}_{2211} \text { ( } \geq 0 \text { by Muirheads's inequality) }
$$

Thus, the strong Four Points Conjecture is verified for the edge-tangential tetrahedra.

Note that the verification of this conjecture which is stronger than A-S conjecture C3 is somewhat simpler (at least for edge-tangential tetrahedra).

\subsection{Trirectangular tetrahedra}

A tetrahedron is called trirectangular if it has a vertex at which all the face angles are right angles. The opposite face to such a vertex we call a base. We label the edge lengths as follows 


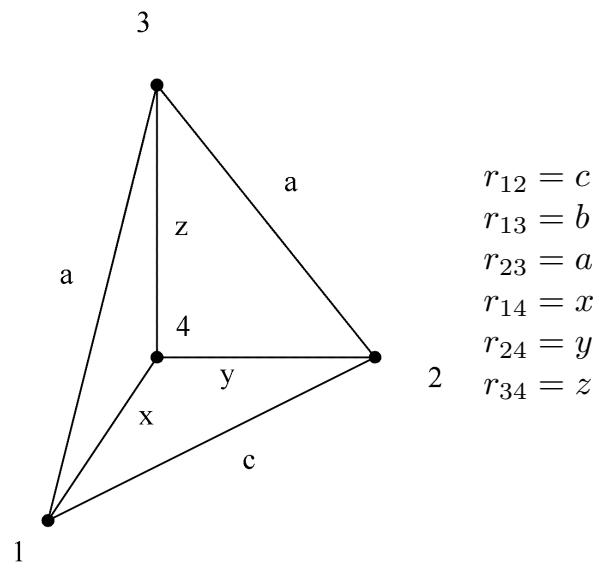

We have following obvious relations: $a^{2}=y^{2}+z^{2}, b^{2}=x^{2}+z^{2}, c^{2}=x^{2}+y^{2}$. By using them we can get

$$
\begin{aligned}
d_{3}(a x, b y, c z) & =2 x y z(a y z+b x z+c x y-a b c) \\
d_{3}(a, b, c) & =2\left(a x^{2}+b y^{2}+c z^{2}-a b c\right) \\
d_{3}(x, y, c) & =2 x y(x+y-c) \\
d_{3}(x, b, z) & =2 x z(x+z-b) \\
d_{3}(a, y, z) & =2 y z(y+z-a)
\end{aligned}
$$

and

$$
\begin{aligned}
& \Re\left(D_{4}\right)-64 a b c x y z-288 V^{2}= \\
& =4 x y z \sum_{c y c} 2 a x^{2}+\sum_{c y c}\left(2 a b+c z+z^{2}\right)(x+y)-10 a b c
\end{aligned}
$$

where $\sum_{c y c}$ has three terms ${ }^{1}$ corresponding to a cycle $((a, x) \rightarrow(b, y) \rightarrow(c, z))$.

By writing $x+y=x+y-c+c$ and using the identity

$$
\sum_{c y c} c^{2} z=\sum_{c y c}\left(x^{2}+y^{2}\right) z=\sum_{c y c}(x+y) z^{2}=\sum_{c y c} z^{2}(x+y-c)+\sum_{c y c} a x^{2}
$$

we get that the second cyclic sum is equal to

$$
\sum_{c y c}\left(2 a b+c z+z^{2}\right)(x+y)=6 a b+\sum_{c y c}\left(2 a b+c z+2 z^{2}\right)(x+y-c)+2 \sum_{c y c} a x^{2}
$$

By inserting this into (2.13) we get

$$
\Re\left(D_{4}\right)-64 a b c x y z-288 V^{2}=4 x y z\left(2 d_{3}(a, b, c)+\sum_{c y c}\left(2 a b+c z+2 z^{2}\right)(x+y-c)\right)
$$

Hence $\Re\left(D_{4}\right) \geq 64 a b c x y z$ so the verification of the C2 of Atiyah-Sutcliffe for trirectangular tetrahedra is finished.

$$
{ }^{1} \sum_{c y c} f(a, b, c, x, y, z)=f(a, b, c, x, y, z)+f(b, c, a, y, z, x)+f(c, a, b, z, x, y)
$$




\subsection{Atiyah-Sutcliffe conjectures for regular and semi-regular tetrahedra}

Semiregular (SR) tetrahedra are one of the simplest configurations of tetrahedra. These tetrahedra have opposite edges equal and hence all faces are congruent. Sometimes semi-regular tetrahedra are called isosceles tetrahedra.

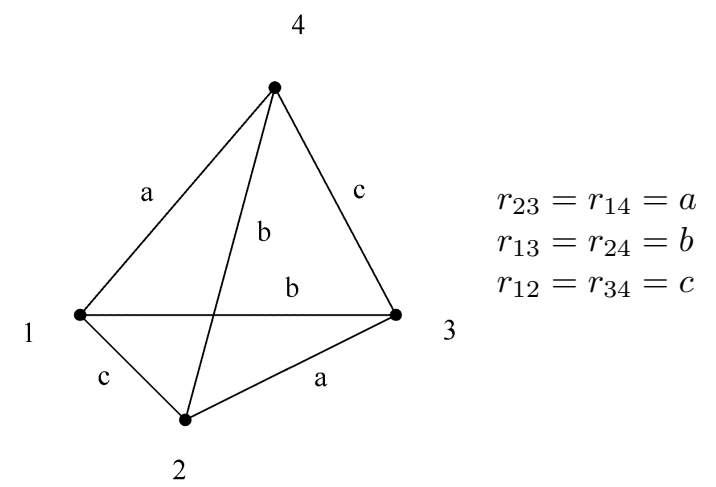

By (2.8) we get

$$
288 V^{2}-4 d_{3}\left(r_{12} r_{34}, r_{13} r_{24}, r_{14} r_{23}\right)=0\left(\Rightarrow 288 V^{2}=4 d_{3}\left(r_{12} r_{34}, r_{13} r_{24}, r_{14} r_{23}\right)\right)
$$

By (2.7) we get

$$
\begin{aligned}
A_{4} & =\sum_{l=1}^{4}\left(d_{3}\left(r_{i l}, r_{j l}, r_{k l}\right)+8 r_{i l} r_{j l} r_{k l}\right) d_{3}\left(r_{i j}, r_{i k}, r_{j k}\right) \\
& =4 d_{3}(a, b, c)^{2}+32 a b c d_{3}(a, b, c)
\end{aligned}
$$

The quantity in the weak Four Points Conjecture is

$$
\begin{aligned}
& \text { l.h.s }- \text { r.h.s }= \\
& =A_{4}-\left(4+\frac{3}{4}\right) 288 V^{2}-\sum_{l=1}^{4}\left(4+\frac{1}{4} \frac{d_{3}\left(r_{i j}, r_{i k}, r_{j k}\right)}{r_{i j} r_{i k} r_{j k}}\right) r_{i l} r_{j l} r_{k l} d_{3}\left(r_{i j}, r_{i k}, r_{j k}\right) \\
& =4 d_{3}(a, b, c)^{2}+32 a b c d_{3}(a, b, c)-(16+3) d_{3}\left(a^{2}, b^{2}, c^{2}\right)-\left[16 a b c d_{3}(a, b, c)+d_{3}(a, b, c)^{2}\right] \\
& =3\left(d_{3}(a, b, c)^{2}-d_{3}\left(a^{2}, b^{2}, c^{2}\right)\right)+16\left(a b c d_{3}(a, b, c)-d_{3}\left(a^{2}, b^{2}, c^{2}\right)\right) \geq 0
\end{aligned}
$$

by using the inequalities $a b c \geq d_{3}(a, b, c)$ and $d_{3}(a, b, c)^{2} \geq d_{3}\left(a^{2}, b^{2}, c^{2}\right)$ (see Appendix 2, Proposition 2.13; also see [19] or [20]).

This proves the weak Four Points Conjecture for semiregular tetrahedra.

We can also prove strong Four Points Conjecture by noting that

$$
\frac{\Re\left(D_{4}\right)}{64 a^{2} b^{2} c^{2}} \geq 1+\frac{1}{8} \sum_{l}\left(4+\frac{1}{4}\right)\left(\delta_{l}-1\right)
$$


(where $\left.\delta_{l}=\frac{d_{3}\left(r_{i j}, r_{i k}, r_{j k}\right)}{r_{i j} r_{i k} r_{j k}}\right)$ is equivalent to

$$
\sum \delta_{l}\left(\delta_{l}-1\right)-\left(\frac{1}{2}+\frac{1}{32}\right)\left(\delta_{l}-1\right)=\sum\left(\delta_{l}-1\right)\left(\delta_{l}-\frac{1}{2}-\frac{1}{32}\right) \geq 0 .
$$

The proof of the strong Four Points Conjecture for semiregular tetrahedra reduces to the positivity of the following expression

$$
4\left(d_{3}(a, b, c)^{2}-d_{3}\left(a^{2}, b^{2}, c^{2}\right)\right)+15\left(a b c d_{3}(a, b, c)-d_{3}\left(a^{2}, b^{2}, c^{2}\right)\right) \geq 0
$$

which is also true by the same argument.

\subsection{Atiyah-Sutcliffe conjectures for parallelograms}

Given a parallelogram with vertices 1, 2, 3 and 4 denote by $a, b$ its side lengths and by $e, f$ its diagonals.

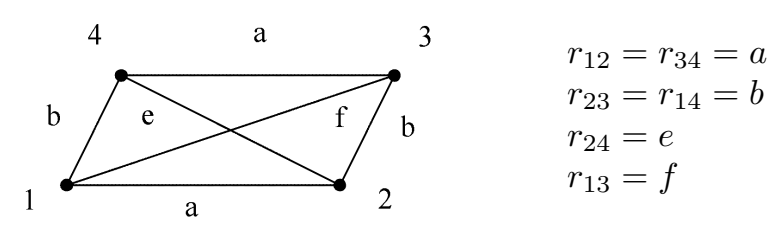

For the numbers $a, b, e, f$ we have the basic relation ("a parallelogram law")

$$
e^{2}+f^{2}=2\left(a^{2}+b^{2}\right)
$$

By using this relation we can rewrite various quantities in the Eastwood-Norbury formula.

Proposition 2.9 We have the following identities

1. $d_{3}(a, b, e)=(a+b-e)(a-b+e)(-a+b+e)=(a+b-e)(a+b-f)(a+b+f)$

2. $\Delta:=(a+b+e) d_{3}(a, b, e)=(a+b+f) d_{3}(a, b, f)=$ $=(a+b+e)(a+b+f)(a+b-e)(a+b-f)=$ $=2 a^{2} b^{2}+2 a^{2} e^{2}+2 b^{2} e^{2}-a^{4}-b^{4}-e^{4}=2 a^{2} b^{2}+2 a^{2} f^{2}+2 b^{2} f^{2}-a^{4}-b^{4}-f^{4}$

3. $4 a b+e^{2}-f^{2}=2(a+b+f)(a+b-f), 4 a b+f^{2}-e^{2}=2(a+b+e)(a+b-e)$

4. $d_{3}\left(a^{2}, b^{2}, e f\right)=\left(a^{2}+b^{2}-e f\right) \Delta$

5. $d_{3}(a, b, e) d_{3}(a, b, f)-d_{3}\left(a^{2}, b^{2}, e f\right)=(2 a b-2 e f-(a+b)(e+f)) \Delta$

6. $e d_{3}(a, b, f)+f d_{3}(a, b, e)=(a+b-e)(a+b-f)\left(e^{2}+f^{2}+(a+b)(e+f)\right)$

7. $\left(4 a b+e^{2}-f^{2}\right) e d_{3}(a, b, f)+\left(4 a b+f^{2}-e^{2}\right) f d_{3}(a, b, e)=2((a+b)(e+f)-$ $2 e f) \Delta$ 


\section{Proof .}

For 1. we write $(a-b+e)(-a+b+e)=e^{2}-(a-b)^{2}=2 a^{2}+2 b^{2}-f^{2}-(a-b)^{2}=$ $(a+b-f)(a+b+f)$. Identity 2. follows from 1. directly. For 3. we substitute $e^{2}=2 a^{2}+2 b^{2}-f^{2}$ and simplify. For 4 . we compute and use $2 .:$

$$
\left(a^{2}-b^{2}+e f\right)\left(-a^{2}+b^{2}+e f\right)=e^{2} f^{2}-\left(a^{2}-b^{2}\right)^{2}=e^{2}\left(2 a^{2}+2 b^{2}-e^{2}\right)+2 a^{2} b^{2}-a^{4}-b^{4}=\Delta
$$

For 5. we first use 1. and then $4:: d_{3}(a, b, e) d_{3}(a, b, f)-d_{3}\left(a^{2}, b^{2}, e f\right)=(a+b+$ $f)(a+b-e)(a+b-f) d_{3}(a, b, f)-\left(a^{2}+b^{2}-e f\right) \Delta=\left[(a+b)^{2}-(a+b)(e+f)+e f\right] \Delta$ $-\left(a^{2}+b^{2}-e f\right) \Delta=[2 a b+2 e f-(a+b)(e+f)] \Delta$

For 6 . we use 1 . twice.

For 7 . we first use 3. and then 2.:

$$
\begin{aligned}
\text { l.h.s. } & \left.=2(a+b+f)(a+b-f) e d_{3}(a, b, f)+2(a+b+e)(a+b-e) f d_{(} a, b, e\right) \\
& =2[(a+b-f) e+(a+b-e) f] \Delta
\end{aligned}
$$

Now we apply Eastwood-Norbury formula (note that $288 \mathrm{~V}^{2}=0, D_{4}=$ real)

$$
D_{4}-64 \prod r_{i j}=-4 d_{3}\left(a^{2}, b^{2}, c^{2}\right)+A_{4}
$$

where

$$
\begin{aligned}
A_{4} & =2\left[d_{3}(a, b, e)+8 a b e+e\left(e^{2}-f^{2}\right)\right] d_{3}(a, b, f)+2\left[d_{3}(a, b, f)+8 a b f+f\left(f^{2}-e^{2}\right)\right] d_{3}(a, b, e) \\
& =I_{0}+I_{1}+I_{2}
\end{aligned}
$$

where

$$
\begin{aligned}
I_{0} & =4 d_{3}(a, b, e) d_{3}(a, b, f) \\
I_{1} & =2\left[4 a b e+e\left(e^{2}-f^{2}\right)\right] d_{3}(a, b, f)+2\left[4 a b f+f\left(f^{2}-e^{2}\right)\right] d_{3}(a, b, e) \\
& =4((a+b)(e+f)-2 e f) \Delta \quad \text { by } 7 .) \\
I_{2} & =2\left[4 a b e d_{3}(a, b, f)+4 a b f d_{3}(a, b, e)\right] \\
& =8 a b(a+b-e)(a+b-f)\left(e^{2}+f^{2}+(a+b)(e+f)\right) \text { (by 6.) }
\end{aligned}
$$

By using 5. we have

$$
\begin{aligned}
D_{4}-64 \prod r_{i j} & =4\left(d_{3}(a, b, e) d_{3}(a, b, f)-d_{3}\left(a^{2}, b^{2}, e f\right)\right)+I_{1}+I_{2} \\
& =4((2 a b+2 e f-(a+b)(e+f)) \Delta+((a+b)(e+f)-2 e f) \Delta)+I_{2} \\
& =8 a b \Delta+I_{2} \geq 0
\end{aligned}
$$

This proves the Atiyah-Sutcliffe conjecture (C2) for parallelograms. The AtiyahSutcliffe conjecture (C3) for parallelograms

$$
D_{4}^{2} \geq\left(d_{3}(a, b, e)+8 a b e\right)^{2}\left(d_{3}(a, b, f)+8 a b f\right)^{2}
$$

is equivalent to the positivity of

$$
D_{4}-d_{3}(a, b, e) d_{3}(a, b, f)-8\left[a b f d_{3}(a, b, e)+a b e d_{3}(a, b, f)\right]-64 a^{2} b^{2} e f \geq 0
$$


but we can prove a stronger statement

$$
\begin{aligned}
& D_{4}-2 d_{3}(a, b, e) d_{3}(a, b, f)-8\left[a b f d_{3}(a, b, e)+a b e d_{3}(a, b, f)\right]-64 a^{2} b^{2} e f= \\
& =8 a b \Delta-2 d_{3}(a, b, e) d_{3}(a, b, f)=2[4 a b-(a+b-e)(a+b-f)] \Delta \geq 0
\end{aligned}
$$

because the triangle inequalities $b<e+a$ and $a<f+b$ imply

$$
(a+b-e)(a+b-f)<2 a \cdot 2 b=4 a b .
$$

Thus we have verified also C3 for parallelograms.

Finally we verify our strong Four Point Conjecture for parallelograms as follows

$$
\begin{aligned}
& D_{4}-64 \prod r_{i j}-\sum_{4}\left(4+\frac{1}{4}\right) r_{i l} r_{j l} r_{k l} d_{3}\left(r_{i j}, r_{j l}, r_{i k}\right) \\
& =8 a b \Delta-\frac{1}{4}\left(I_{2} / 4\right) \\
& =8 a b(a+b-e)(a+b-f)\left[(a+b+e)(a+b+f)-\frac{1}{16}\left(e^{2}+f^{2}+(a+b)(e+f)\right)\right] \\
& =\frac{1}{2} a b(a+b-e)(a+b-f)\left[16\left((a+b)^{2}+(a+b)(e+f)+e f\right)-\left(2 a^{2}+2 b^{2}+(a+b)(e+f)\right)\right] \\
& =\frac{1}{2} a b(a+b-e)(a+b-f)\left[14\left(a^{2}+b^{2}\right)+32 a b+15(a+b)(e+f)+16 e f\right] \geq 0
\end{aligned}
$$

\subsection{Atiyah-Sutcliffe conjectures for "wedge" tetrahedra}

A tetrahedron with two pairs of opposite edges having the same length we simply call a "wedge" tetrahedron.

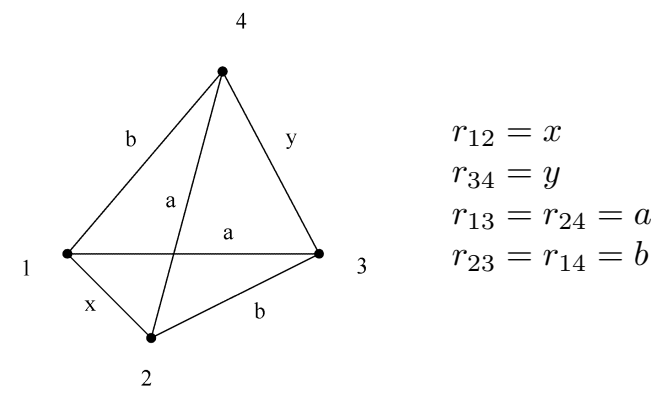

If $x=y=c$ we get a semiregular tetrahedron and if all points lie in a plane then we get either a parallelogram or an isosceles trapezium.

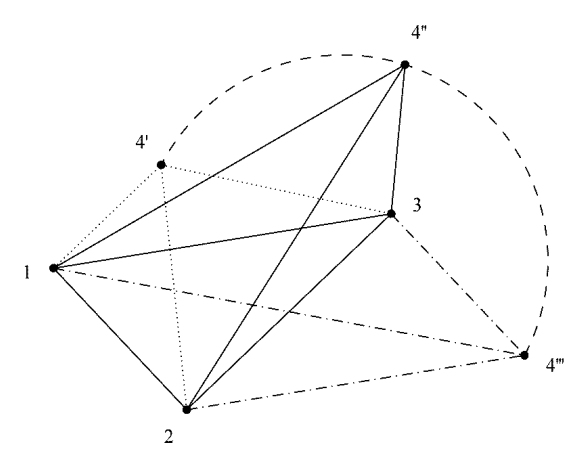


Again we compute the data appearing in the Eastwood-Norbury formula

$$
\begin{aligned}
& -4 d_{3}\left(r_{12} r_{34}, r_{13} r_{24}, r_{14} r_{23}\right)= \\
& =-4 d_{3}\left(x y, a^{2}, b^{2}\right) \\
& =-4\left(x y-a^{2}+b^{2}\right)\left(x y+a^{2}-b^{2}\right)\left(a^{2}+b^{2}-x y\right)
\end{aligned}
$$

and we have the basic inequalities

$$
x y+b^{2} \geq a^{2}, x y+a^{2} \geq b^{2}, a^{2}+b^{2} \geq x y
$$

The positivity of the volume

$$
144 V^{2}=\left(x y-a^{2}+b^{2}\right)\left(x y+a^{2}-b^{2}\right)\left(2 a^{2}+2 b^{2}-x^{2}-y^{2}\right) \quad\left(\leq 2 d_{3}\left(a^{2}, b^{2}, x y\right)\right)
$$

gives us one more basic inequality

$$
2 a^{2}+2 b^{2} \geq x^{2}+y^{2}
$$

We have

$$
\begin{aligned}
A_{4}= & 2\left[a\left((b+x)^{2}-a^{2}\right)+b\left((a+x)^{2}-b^{2}\right)+x\left((a+b)^{2}-y^{2}\right)\right] d_{3}(a, b, y) \\
& +2\left[a\left((b+y)^{2}-a^{2}\right)+b\left((a+y)^{2}-b^{2}\right)+y\left((a+b)^{2}-x^{2}\right)\right] d_{3}(a, b, x)
\end{aligned}
$$

By using identity

$$
d_{3}(a, b, c)=a\left(b^{2}+c^{2}-a^{2}\right)+b\left(a^{2}+c^{2}-b^{2}\right)+c\left(a^{2}+b^{2}-c^{2}\right)-2 a b c
$$

we can rewrite $A_{4}$ as follows

$$
\begin{aligned}
A_{4}= & 2\left[4 a b x+d_{3}(a, b, x)-x\left(a^{2}+b^{2}-x^{2}\right)+2 a b x+x\left((a+b)^{2}-y^{2}\right)\right] d_{3}(a, b, y) \\
& +2\left[4 a b y+d_{3}(a, b, y)-y\left(a^{2}+b^{2}-y^{2}\right)+2 a b y+y\left((a+b)^{2}-x^{2}\right)\right] d_{3}(a, b, x) \\
= & 2\left[4 a b x+d_{3}(a, b, x)-x\left((a-b)^{2}-x^{2}\right)+x\left((a+b)^{2}-y^{2}\right)\right] d_{3}(a, b, y) \\
& +2\left[4 a b y+d_{3}(a, b, y)-y\left((a-b)^{2}-y^{2}\right)+y\left((a+b)^{2}-x^{2}\right)\right] d_{3}(a, b, x) \\
= & \left\{8 a b x+d_{3}(a, b, x)+\left[d_{3}(a, b, x)-2 x\left((a-b)^{2}-x^{2}\right)\right]+2 x\left((a+b)^{2}-y^{2}\right)\right\} d_{3}(a, b, y) \\
& +\left\{8 a b y+d_{3}(a, b, y)+\left[d_{3}(a, b, y)-2 y\left((a-b)^{2}-y^{2}\right)\right]+2 y\left((a+b)^{2}-x^{2}\right)\right\} d_{3}(a, b, x)
\end{aligned}
$$

Now we compute

$$
\begin{aligned}
& d_{3}(a, b, x)-2 x\left((a-b)^{2}-x^{2}\right)= \\
& =(a+b-x)(a-b+x)(-a+b+x)+2 x(a-b+x)(-a+b+x) \\
& =(a+b+x)(a-b+x)(-a+b+x)
\end{aligned}
$$

The contribution $A_{4}^{[]}$of both square brackets in $A_{4}$ is equal to

$$
\begin{aligned}
A_{4}^{[]]}:= & {\left[d_{3}(a, b, x)-2 x\left((a-b)^{2}-x^{2}\right)\right] d_{3}(a, b, y)+} \\
& +\left[d_{3}(a, b, y)-2 y\left((a-b)^{2}-y^{2}\right)\right] d_{3}(a, b, x) \\
= & \left(x^{2}-(a-b)^{2}\right)\left(y^{2}-(a-b)^{2}\right)[(a+b+x)(a+b-y)+(a+b+y)(a+b-x)] \\
= & \left(x^{2}-(a-b)^{2}\right)\left(y^{2}-(a-b)^{2}\right)\left(2(a+b)^{2}-2 x y\right) \\
= & 4 a b\left(x^{2}-(a-b)^{2}\right)\left(y^{2}-(a-b)^{2}\right)+2\left(x^{2}-(a-b)^{2}\right)\left(y^{2}-(a-b)^{2}\right)\left(a^{2}+b^{2}-x y\right)
\end{aligned}
$$


At this point we have discovered the following beautiful identity

$$
\begin{aligned}
& {\left[x^{2}-(a-b)^{2}\right]\left[y^{2}-(a-b)^{2}\right]=} \\
& =\left(x y-a^{2}+b^{2}\right)\left(x y+a^{2}-b^{2}\right)+(a-b)^{2}\left(2 a^{2}+2 b^{2}-x^{2}-y^{2}\right)
\end{aligned}
$$

By this identity we can write

$$
\begin{aligned}
A_{4}^{[]}= & 4 a b\left(x^{2}-(a-b)^{2}\right)\left(y^{2}-(a-b)^{2}\right) \\
& +2(a-b)^{2}\left(2 a^{2}+2 b^{2}-x^{2}-y^{2}\right)\left(a^{2}+b^{2}-x y\right) \\
& +2 d_{3}\left(a^{2}, b^{2}, x y\right)
\end{aligned}
$$

Lemma 2.10 We have the following inequality for "wedge" tetrahedra

$$
d_{3}\left(a^{2}, b^{2}, x y\right) \leq 2 a b\left(x^{2}-(a-b)^{2}\right)\left(y^{2}-(a-b)^{2}\right)
$$

Proof .

Recall that

$$
d_{3}\left(a^{2}, b^{2}, x y\right)=\left(a^{2}+b^{2}-x y\right)\left(a^{2}-b^{2}+x y\right)\left(-a^{2}+b^{2}+x y\right)
$$

Let $a \geq b$. Then the triangle inequalities $a \leq b+x$ and $a \leq b+y$ imply $(a-b)^{2} \leq x y$ i.e. $a^{2}+b^{2}-x y \leq 2 a b$. Since $2 a^{2}+2 b^{2}-x^{2}-y^{2} \geq 0$ (inequality $(2.19))$ then from our inequality (2.24) it follows that

$$
\left(a^{2}-b^{2}+x y\right)\left(-a^{2}+b^{2}+x y\right) \leq\left(x^{2}-(a-b)^{2}\right)\left(y^{2}-(a-b)^{2}\right)
$$

By multiplying the last two inequalities Lemma follows.

As a consequence of Lemma we get immediately that

$$
A_{4} \geq A_{4}^{[]]} \geq 4 d_{3}\left(a^{2}, b^{2}, x y\right)
$$

because the remaining terms in $A_{4}$ are all nonnegative. This verifies the A-S conjecture $\mathrm{C} 2$ for "wedge" tetrahedra.

Remark 2.11 Instead of splitting $2(a+b)^{2}-2 x y=4 a b+2\left(a^{2}+b^{2}-x y\right)$ (used above), we can use the identity

$$
2(a+b)^{2}-2 x y=4\left(a^{2}+b^{2}-x y\right)+2\left(x y-(a-b)^{2}\right)
$$

to obtain explicit formula for $A_{4}^{[]}$:

$$
\begin{aligned}
& A_{4}^{[]}= \\
& {\left[4\left(a^{2}+b^{2}-x y\right)+2\left(x y-(a-b)^{2}\right)\right]\left[\left(x y-a^{2}+b^{2}\right)\left(x y+a^{2}-b^{2}\right)+\right.} \\
& \left.+(a-b)^{2}\left(2 a^{2}+2 b^{2}-x^{2}-y^{2}\right)\right]= \\
& =4 d_{3}\left(a^{2}, b^{2}, x y\right)+4\left(a^{2}+b^{2}-x y\right)\left(2 a^{2}+2 b^{2}-x^{2}-y^{2}\right)(a-b)^{2}+ \\
& +2\left(x y-(a-b)^{2}\right)\left(x^{2}-(a-b)^{2}\right)\left(y^{2}-(a-b)^{2}\right)
\end{aligned}
$$

which, without using Lemma 2.10, implies inequality

$$
A_{4}^{[]]} \geq 4 d_{3}\left(a^{2}, b^{2}, x y\right)
$$

needed for the verification of A-S conjecture C2 for "wedge" tetrahedra. 
Now we state a final formula for "wedge" tetrahedra:

$$
\begin{aligned}
\Re\left(D_{4}\right)= & \text { First explicit formula for wedge tetrahedra: } \\
& \left(d_{3}(a, b, x)+8 a b x\right)\left(d_{3}(a, b, y)+8 a b y\right)+d_{3}(a, b, x) d_{3}(a, b, y)+ \\
& +2 x\left((a+b)^{2}-y^{2}\right) d_{3}(a, b, y)+2 y\left((a+b)^{2}-x^{2}\right) d_{3}(a, b, x)+ \\
& +4\left(a^{2}+b^{2}-x y\right)\left(2 a^{2}+2 b^{2}-x^{2}-y^{2}\right)(a-b)^{2}+ \\
& +2\left(x y-(a-b)^{2}\right)\left(x^{2}-(a-b)^{2}\right)\left(y^{2}-(a-b)^{2}\right) \\
& +288 V^{2}
\end{aligned}
$$

which implies a strengthened A-S conjecture C3 for wedge tetrahedra

$$
\begin{aligned}
\Re\left(D_{4}\right) & \geq\left(d_{3}(a, b, x)+8 a b x\right)\left(d_{3}(a, b, y)+8 a b y\right)+d_{3}(a, b, x) d_{3}(a, b, y)+288 V^{2} \\
\geq & \left(d_{3}(a, b, x)+8 a b x\right)\left(d_{3}(a, b, y)+8 a b y\right)
\end{aligned}
$$

In the sequel we obtain an alternative formula for the real part of the Atiyah determinant for a wedge tetrahedra.

We group terms in $A_{4}$ differently as follows:

$$
\begin{aligned}
A_{4}= & 2\left[4 a b x+d_{3}(a, b, x)+x\left(4 a b+x^{2}-y^{2}\right)\right] d_{3}(a, b, y)+ \\
& +2\left[4 a b y+d_{3}(a, b, y)+x\left(4 a b+y^{2}-x^{2}\right)\right] d_{3}(a, b, x)
\end{aligned}
$$

By letting

$$
2 a^{2}+2 b^{2}-x^{2}-y^{2}=: 2 h \quad(\geq 0)
$$

we can rewrite

$$
4 a b+x^{2}-y^{2}=4 a b+x^{2}+\left(2 h+x^{2}-2 a^{2}-2 b^{2}\right)=2\left(h+x^{2}-(a-b)^{2}\right)
$$

and similarly for

$$
4 a b+y^{2}-x^{2}=2\left(h+y^{2}-(a-b)^{2}\right)
$$

Thus

$$
\begin{aligned}
A_{4}= & 4 d_{3}(a, b, x) d_{3}(a, b, y)+8 a b x d_{3}(a, b, y)+8 a b y d_{3}(a, b, x)+ \\
& +4 h\left(x d_{3}(a, b, y)+y d_{3}(a, b, x)\right)+4 A_{4}^{\prime}
\end{aligned}
$$

where

$$
\begin{aligned}
A_{4}^{\prime} & =x\left(x^{2}-(a-b)^{2}\right) d_{3}(a, b, y)+y\left(y^{2}-(a-b)^{2}\right) d_{3}(a, b, x) \\
& =\left(x^{2}-(a-b)^{2}\right)\left(y^{2}-(a-b)^{2}\right)[x(a+b-y)+y(a+b-x)] \\
& =\left(x^{2}-(a-b)^{2}\right)\left(y^{2}-(a-b)^{2}\right)\left[(x-y)^{2}+x(a+b-x)+y(a+b-y)\right] \\
& =\left[\left(x y-a^{2}+b^{2}\right)\left(x y+a^{2}-b^{2}\right)+2(a-b)^{2} h\right]\left[(x-y)^{2}+x(a+b-x)+y(a+b-y)\right]
\end{aligned}
$$

by our identity (2.24).

Note that

$$
-144 V^{2}+2 d_{3}\left(a^{2}, b^{2}, x y\right)=\left(x y-a^{2}+b^{2}\right)\left(x y+a^{2}-b^{2}\right)(x-y)^{2}
$$


So

$$
\begin{aligned}
A_{4}^{\prime}= & \left(2 d_{3}\left(a^{2}, b^{2}, x y\right)-144 V^{2}\right)+2(a-b)^{2} h[x(a+b-y)+y(a+b-x)] \\
& +\left(x y-a^{2}+b^{2}\right)\left(x y+a^{2}-b^{2}\right)(x(a+b-x)+y(a+b-y))
\end{aligned}
$$

By writing

$$
\begin{aligned}
& 4 A_{4}^{\prime}=2 A_{4}^{\prime}+2 A_{4}^{\prime}= \\
& =2\left(x^{2}-(a-b)^{2}\right)\left(y^{2}-(a-b)^{2}\right)[x(a+b-y)+y(a+b-x)]+ \\
& +\left\{4 d_{3}\left(a^{2}, b^{2}, x y\right)-288 V^{2}+4(a-b)^{2} h[x(a+b-y)+y(a+b-x)]+\right. \\
& \left.+2\left(x y-a^{2}+b^{2}\right)\left(x y+a^{2}-b^{2}\right)(x(a+b-x)+y(a+b-y))\right\}= \\
& =4 d_{3}\left(a^{2}, b^{2}, x y\right)-288 V^{2}+\left[2\left(x^{2}-(a-b)^{2}\right)\left(y^{2}-(a-b)^{2}\right)+4(a-b)^{2} h\right] . \\
& \cdot(x(a+b-y)+y(a+b-x))+ \\
& +2\left(x y-a^{2}+b^{2}\right)\left(x y+a^{2}-b^{2}\right)[x(a+b-x)+y(a+b-y)]
\end{aligned}
$$

we obtain the following explicit formula for the real part of Atiyah determinant for "wedge" tetrahedron:

$$
\begin{aligned}
\Re\left(D_{4}\right)= & \frac{\text { Second explicit formula for wedge tetrahedra: }}{\left(d_{3}(a, b, x)+8 a b x\right)\left(d_{3}(a, b, y)+8 a b y\right)+3 d_{3}(a, b, x) d_{3}(a, b, y)+} \\
& +2 x\left((a+b)^{2}-y^{2}\right) d_{3}(a, b, y)+2 y\left((a+b)^{2}-x^{2}\right) d_{3}(a, b, x)+ \\
& 2\left(x^{2} y^{2}-\left(a^{2}-b^{2}\right)\right)[x(a+b-x)+y(a+b-y)]+ \\
& +2\left[(a-b)^{2}(x(a+b-y)+y(a+b-x))\right]\left(2 a^{2}+2 b^{2}-x^{2}-y^{2}\right)
\end{aligned}
$$

which implies another strengthening of the Atiyah-Sutcliffe conjecture C3 for "wedge" tetrahedra

$$
\begin{aligned}
\Re\left(D_{4}\right) & \geq\left(d_{3}(a, b, x)+8 a b x\right)\left(d_{3}(a, b, y)+8 a b y\right)+3 d_{3}(a, b, x) d_{3}(a, b, y) \\
& \geq\left(d_{3}(a, b, x)+8 a b x\right)\left(d_{3}(a, b, y)+8 a b y\right)
\end{aligned}
$$

\subsection{Atiyah determinant for triangles and quadrilaterals via trigonometry}

Denote the three points $x_{1}, x_{2}, x_{3}$ simply by symbols $1,2,3$ and let $X, Y$ and $Z$ denote the angles of the triangle at vertices 1, 2 and 3 respectively. Then we can express the Atiyah determinant $D_{3}=d_{3}\left(r_{12}, r_{13}, r_{23}\right)+8 r_{12} r_{13} r_{23}$ as follows

$$
D_{3}=4 r_{12} r_{13} r_{23}\left(\cos ^{2} \frac{X}{2}+\cos ^{2} \frac{Y}{2}+\cos ^{2} \frac{Z}{2}\right) .
$$

This follows, by using cosine law and sum to product formula for cosine, from the following identity

$$
\begin{aligned}
d_{3}(a, b, c)+8 a b c & =(a+b-c)(a-b+c)(-a+b+c)+8 a b c \\
& =a\left((b+c)^{2}-a^{2}\right)+b\left((c+a)^{2}-b^{2}\right)+c\left((a+b)^{2}-c^{2}\right) .
\end{aligned}
$$

Now we shall translate the Eastwood-Norbury formula for (planar quadrilaterals) into a trigonometric form. Denote the four points $x_{1}, x_{2}, x_{3}, x_{4}$ simply by symbols $1,2,3,4$ and denote by

$$
\left(X^{(1)}, Y^{(1)}, Z^{(1)}\right), \quad\left(X^{(2)}, Y^{(2)}, Z^{(2)}\right), \quad\left(X^{(3)}, Y^{(3)}, Z^{(3)}\right), \quad\left(X^{(4)}, Y^{(4)}, Z^{(4)}\right)
$$


the angles of the triangles $234,341,412,123$ in this cyclic order (i.e. the angle of a triangle 412 at vertex 2 is $Z^{(3)}$ etc.).

Next we denote by $c_{l},(1 \leq l \leq 4)$, the sums of cosines squared of half-angles of the $l$-th triangle i.e.:

$$
c_{l}:=\cos ^{2} \frac{X^{(l)}}{2}+\cos ^{2} \frac{Y^{(l)}}{2}+\cos ^{2} \frac{Z^{(l)}}{2}, \quad l=1,2,3,4 .
$$

Similarly, we denote by $\widehat{c}_{l},(1 \leq l \leq 4)$, the sum of cosines squared of half-angles at the $l$-th vertex of our quadrilateral thus

$$
\begin{aligned}
& \widehat{c}_{1}=\cos ^{2} \frac{Z^{(2)}}{2}+\cos ^{2} \frac{Y^{(3)}}{2}+\cos ^{2} \frac{X^{(4)}}{2} \\
& \widehat{c}_{2}=\cos ^{2} \frac{Z^{(3)}}{2}+\cos ^{2} \frac{Y^{(4)}}{2}+\cos ^{2} \frac{X^{(1)}}{2} \\
& \widehat{c}_{3}=\cos ^{2} \frac{Z^{(4)}}{2}+\cos ^{2} \frac{Y^{(1)}}{2}+\cos ^{2} \frac{X^{(2)}}{2} \\
& \widehat{c}_{4}=\cos ^{2} \frac{Z^{(1)}}{2}+\cos ^{2} \frac{Y^{(2)}}{2}+\cos ^{2} \frac{X^{(3)}}{2}
\end{aligned}
$$

Then the term $A_{4}$ in the Eastwood-Norbury formula can be rewritten as

$$
\begin{aligned}
A_{4} & =\sum_{l=1}^{4}\left(4 r_{l i} r_{l j} r_{l k} \widehat{c}_{l}\right) \cdot 4 r_{i j} r_{i k} r_{j k}\left(c_{l}-2\right) \\
& =16 r_{12} r_{13} r_{23} r_{14} r_{24} r_{34} \sum_{l=1}^{4} \widehat{c}_{l}\left(c_{l}-2\right) .
\end{aligned}
$$

where for each $l$ we write $\{1,2,3,4\} \backslash\{l\}=\{i<j<k\}$.

In order to rewrite the term $-4 d_{3}\left(r_{12} r_{34}, r_{13} r_{24}, r_{14} r_{23}\right)$ into a trigonometric form we recall a theorem of Möbius ([12]) which claims that for any quadrilateral 1234 in a plane the products $r_{12} r_{34}, r_{13} r_{24}$ and $r_{14} r_{23}$ are proportional to the sides of a triangle whose angles are the differences of angles in the quadrilateral 1234:

$$
\begin{aligned}
& X=\varangle 134-\varangle 124 \\
& Y=\varangle 214-\varangle 234 \\
& Z=\varangle 413-\varangle 423
\end{aligned}
$$

Thus

$$
-4 d_{3}\left(r_{12} r_{34}, r_{13} r_{24}, r_{14} r_{23}\right)=-16 r_{12} r_{13} r_{23} r_{14} r_{24} r_{34}(c-2)
$$

where

$$
c=\cos ^{2} \frac{X}{2}+\cos ^{2} \frac{Y}{2}+\cos ^{2} \frac{Z}{2} .
$$


Thus we have obtained a trigonometric formula for Atiyah determinant of quadrilaterals

$$
\begin{aligned}
\Re\left(D_{4}\right) & =\prod_{1 \leq i<j \leq 4} r_{i j}\left(64-16(c-2)+16 \sum_{l=1}^{4} \widehat{c}_{l}\left(c_{l}-2\right)\right) \\
& =16 \prod_{1 \leq i<j \leq 4} r_{i j}\left(6-c+\sum_{l=1}^{4} \widehat{c}_{l}\left(c_{l}-2\right)\right)
\end{aligned}
$$

Now we shall verify Atiyah-Sutcliffe conjecture for cyclic quadrilaterals.

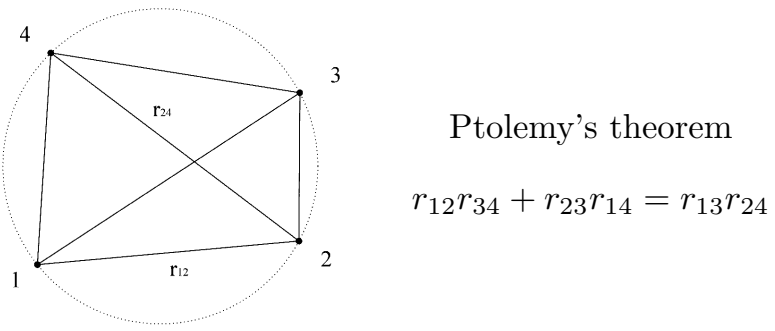

In this case, by a well known Ptolemy's theorem, we see that

$$
-4 d_{3}\left(r_{12} r_{34}, r_{13} r_{24}, r_{14} r_{23}\right)=0 \quad(\Leftrightarrow c=2)
$$

By using the equality of angles $Z^{(2)}=X^{(1)}, Z^{(3)}=X^{(2)}, Z^{(4)}=X^{(3)}, Z^{(1)}=$ $X^{(4)}$ and $Y^{(1)}+Y^{(3)}=\pi=Y^{(2)}+Y^{(4)}$ (angles with vertex on a circle's circumference with the same endpoints are equal or supplement of each other)we obtain

$$
\begin{aligned}
& \widehat{c}_{1}=\cos ^{2} \frac{X^{(1)}}{2}+\sin ^{2} \frac{Y^{(1)}}{2}+\cos ^{2} \frac{Z^{(1)}}{2}=c_{1}-\cos Y^{(1)}, \\
& \widehat{c}_{2}=\cos ^{2} \frac{X^{(2)}}{2}+\sin ^{2} \frac{Y^{(2)}}{2}+\cos ^{2} \frac{Z^{(2)}}{2}=c_{2}-\cos Y^{(2)}, \\
& \widehat{c}_{3}=\cos ^{2} \frac{X^{(3)}}{2}+\sin ^{2} \frac{Y^{(3)}}{2}+\cos ^{2} \frac{Z^{(3)}}{2}=c_{3}-\cos Y^{(3)}, \\
& \widehat{c}_{4}=\cos ^{2} \frac{X^{(4)}}{2}+\sin ^{2} \frac{Y^{(4)}}{2}+\cos ^{2} \frac{Z^{(4)}}{2}=c_{4}-\cos Y^{(4)} .
\end{aligned}
$$

Now we have

$$
\begin{aligned}
\Re\left(D_{4}\right) & =\left(\prod_{1 \leq i<j \leq 4} r_{i j}\right)\left(64+16 \sum_{l=1}^{4}\left(c_{l}-\cos Y^{(l)}\right)\left(c_{l}-2\right)\right) \\
& \geq\left(\prod_{1 \leq i<j \leq 4} r_{i j}\right)\left(64+16 \sum_{l=1}^{4}\left(c_{l}-1\right)\left(c_{l}-2\right)\right)
\end{aligned}
$$


(here we have used that $2 \leq c_{l}\left(\leq \frac{9}{4}\right)$ for each $l=1,2,3,4$ )

$$
\begin{aligned}
& \geq\left(\prod_{1 \leq i<j \leq 4} r_{i j}\right)\left(64+16 \sum_{l=1}^{4}\left(c_{l}-2\right)+16 \sum_{l=1}^{4}\left(c_{l}-2\right)^{2}\right) \\
& \geq\left(\prod_{1 \leq i<j \leq 4} r_{i j}\right)\left(64+16 \sum_{l=1}^{4}\left(c_{l}-2\right)+4\left(\sum_{l=1}^{4}\left(c_{l}-2\right)\right)^{2}\right)
\end{aligned}
$$

(by quadratic-arithmetic inequality)

$$
\begin{aligned}
& =\left(\prod_{1 \leq i<j \leq 4} r_{i j}\right)\left(\left(8+\sum_{l=1}^{4}\left(c_{l}-2\right)\right)^{2}+3\left(\sum_{l=1}^{4}\left(c_{l}-2\right)\right)^{2}\right) \\
& =\left(\prod_{1 \leq i<j \leq 4} r_{i j}\right)\left(\left(\sum_{l=1}^{4} c_{l}\right)^{2}+3\left(\sum_{l=1}^{4}\left(c_{l}-2\right)\right)^{2}\right) \\
& \geq\left(\prod_{1 \leq i<j \leq 4} r_{i j}\right)\left(\sum_{l=1}^{4} c_{l}\right)^{2} \geq 16 \sqrt{c_{1} c_{2} c_{3} c_{4}} \prod_{1 \leq i<j \leq 4} r_{i j}
\end{aligned}
$$

by $\mathrm{A}-\mathrm{G}$ inequality.

Finally,

$$
\begin{aligned}
\left|D_{4}\right|^{2} & =\left|\Re\left(D_{4}\right)\right|^{2} \geq 4^{4} c_{1} c_{2} c_{3} c_{4} \prod_{1 \leq i<j \leq 4} r_{i j}^{2} \\
& =\prod_{l=1}^{4}\left(4 r_{i j} r_{i k} r_{j k} c_{l}\right)=\prod_{l=1}^{4}\left(d_{3}\left(r_{i j}, r_{i k}, r_{j k}\right)+8 r_{i j} r_{i k} r_{j k}\right)
\end{aligned}
$$

where for each $l$ we write $\{1,2,3,4\} \backslash\{l\}=\{i<j<k\}$. This finishes verification of Atiyah-Sutcliffe conjectures for cyclic quadrilaterals.

Proposition 2.12 The weak Four Points Conjecture for cyclic quadrilaterals holds true.

Proof .

From the formula obtained above we proceed along a different path 


$$
\begin{aligned}
\Re\left(D_{4}\right) & =\left(\prod_{1 \leq i<j \leq 4} r_{i j}\right)\left(64+16 \sum_{l=1}^{4}\left(c_{l}-\cos Y^{(l)}\right)\left(c_{l}-2\right)\right) \\
& =\left(\prod_{1 \leq i<j \leq 4} r_{i j}\right)\left(4 \sum_{l=1}^{4}\left[4+4\left(c_{l}-\cos Y^{(l)}\right)\left(c_{l}-2\right)\right]\right) \\
& =\left(\prod_{1 \leq i<j \leq 4} r_{i j}\right)\left(4 \sum_{l=1}^{4}\left[c_{l}^{2}+\left(c_{l}-2\right)\left[3\left(c_{l}-2\right)+4\left(1-\cos Y^{(l)}\right)\right]\right]\right) \\
& \geq\left(\prod_{1 \leq i<j \leq 4} r_{i j}\right)\left(4 \sum_{l=1}^{4} c_{l}^{2}\right)\left(\text { because } 2 \leq c_{l} \text { for each } l=1,2,3,4\right) \\
& =\prod r_{i j}\left(\frac{1}{4} \sum_{l=1}^{4}\left(\frac{d_{3}\left(r_{i j}, r_{i k}, r_{j k}\right)+8 r_{i j} r_{i k} r_{j k}}{r_{i j} r_{i k} r_{j k}}\right)^{2}\right)
\end{aligned}
$$

and this verifies the weak Four Points Conjecture for cyclic quadrilaterals.

\subsection{Verification of the Strong four points conjecture for a triangle with orthocenter}

Let $1,2,3,4$ be the points so that $4=$ orthocenter of the triangle $\triangle 123$ and let $X, Y, Z$ denote the angles of the triangle $\triangle 123$ at vertices $1,2,3$ respectively. Note that $\angle 243=\pi-X, \angle 341=\pi-Y, \angle 142=\pi-Z$. Then, using the notations of (2.8), we have the following formula for the normalized Atiyah determinant

$$
\mathcal{D}_{4}=\frac{D_{4}}{64 \prod_{1 \leq i<j \leq 4} r_{i j}}=1-\frac{1}{4}(c-2)+\frac{1}{4} \sum_{l=1}^{4} \widehat{c}_{l}\left(c_{l}-2\right)
$$

where

$$
\begin{aligned}
& c=\cos ^{2} \frac{\angle 243-\angle 213}{2}+\cos ^{2} \frac{\angle 341-\angle 321}{2}+\cos ^{2} \frac{\angle 142-\angle 132}{2} \\
& =\sin ^{2} X+\sin ^{2} Y+\sin ^{2} Z \\
& c_{1}=\cos ^{2} \frac{\frac{\pi}{2}-Z}{2}+\cos ^{2} \frac{\frac{\pi}{2}-Y}{2}+\cos ^{2} \frac{\pi-X}{2}=\frac{3-\cos X+\sin Y+\sin Z}{2} \\
& c_{2}=\frac{3+\sin X-\cos Y+\sin Z}{2}, \quad c_{3}=\frac{3+\sin X+\sin Y-\cos Z}{2} \\
& c_{4}=\cos ^{2} \frac{X}{2}+\cos ^{2} \frac{Y}{2}+\cos ^{2} \frac{Z}{2}=\frac{3+\cos X+\cos Y+\cos Z}{2} \\
& \widehat{c}_{1}=\cos ^{2} \frac{X}{2}+\cos ^{2} \frac{\frac{\pi}{2}-Y}{2}+\cos ^{2} \frac{\frac{\pi}{2}-Z}{2}=\frac{3+\cos X+\sin Y+\sin Z}{2} \\
& \widehat{c}_{2}=\frac{3+\sin X+\cos Y+\sin Z}{2}, \quad \widehat{c}_{3}=\frac{3+\sin X+\sin Y+\cos Z}{2} \\
& \widehat{c}_{4}=\cos ^{2} \frac{\pi-X}{2}+\cos ^{2} \frac{\pi-Y}{2}+\cos ^{2} \frac{\pi-Z}{2}=\frac{3-\cos X-\cos Y-\cos Z}{2}
\end{aligned}
$$


Now we compute

$$
\begin{aligned}
\left(\widehat{c}_{1}-1\right)\left(c_{1}-2\right)-\frac{c-2}{4}= & \frac{1+\cos X+\sin Y+\sin Z}{2} \cdot \frac{-1-\cos X+\sin Y+\sin Z}{2}- \\
& -\frac{1}{4}\left(\sin ^{2} X+\sin ^{2} Y+\sin ^{2} Z-2\right)= \\
= & -\frac{\cos X}{2}+\frac{\sin Y \sin Z}{2}=\frac{\cos Y \cos Z}{2} \\
\left(\widehat{c}_{2}-1\right)\left(c_{2}-2\right)-\frac{c-2}{4}= & -\frac{\cos Y}{2}+\frac{\sin X \sin Z}{2}=\frac{\cos X \cos Z}{2} \\
\left(\widehat{c}_{3}-1\right)\left(c_{3}-2\right)-\frac{c-2}{4}= & -\frac{\cos Z}{2}+\frac{\sin X \sin Y}{2}=\frac{\cos X \cos Y}{2} \\
\left(\widehat{c}_{4}-1\right)\left(c_{4}-2\right)-\frac{c-2}{4}= & \frac{1-\cos X-\cos Y-\cos Z}{2} \cdot \frac{\cos X+\cos Y+\cos Z-1}{2}- \\
& -\frac{1}{4}\left(\sin ^{2} X+\sin ^{2} Y+\sin ^{2} Z-2\right) \\
= & -\frac{1}{2}+\frac{1}{2}(\cos X+\cos Y+\cos Z)- \\
& -\frac{1}{2}(\cos X \cos Y+\cos X \cos Z+\cos Y \cos Z)
\end{aligned}
$$

By adding last four equations we get

$$
\begin{aligned}
& \sum_{l=1}^{4}\left(\widehat{c}_{l}-1\right)\left(c_{l}-2\right)-(c-2)= \\
& \quad=-\frac{1}{2}+\frac{1}{2}(\cos X+\cos Y+\cos Z) \\
& \quad=c_{4}-2
\end{aligned}
$$

Claim:

$$
c_{4} \geq \frac{1}{4}\left(c_{1}+c_{2}+c_{3}+c_{4}\right)
$$

Proof of claim: It is equivalent to the inequality (for angles of any triangle)

$$
2(\cos X+\cos Y+\cos Z) \geq \sin X+\sin Y+\sin Z
$$

We can suppose that $0 \leq X, Y \leq \pi / 2$. Then $Z=\pi-X-Y$. The identity

$$
\begin{aligned}
& 2(\cos X+\cos Y-\cos (X+Y))-\sin X-\sin Y-\sin (X+Y)= \\
& =2(1-\sin X)(1-\sin Y)+(1-\cos X)(\sin Y+\cos Y-1)+ \\
& \quad+(1-\cos Y)(\sin X+\cos X-1) \\
& \geq 0
\end{aligned}
$$

proves the Claim directly (The inequality $\left(^{*}\right)$ can be deduced also from a Blundon's fundamental inequality).

By combining (2.26), (2.27) and (2.28) we obtain

$$
\mathcal{D}_{4} \geq 1+\frac{1}{4} \sum_{l=1}^{4}\left(1+\frac{1}{4}\right)\left(c_{l}-2\right)
$$

what verifies the strong four points Conjecture for a four point configuration of vertices and the orthocenter of any triangle. 
Svrtan, Urbiha Atiyah-Sutcliffe conjectures for four points configurations

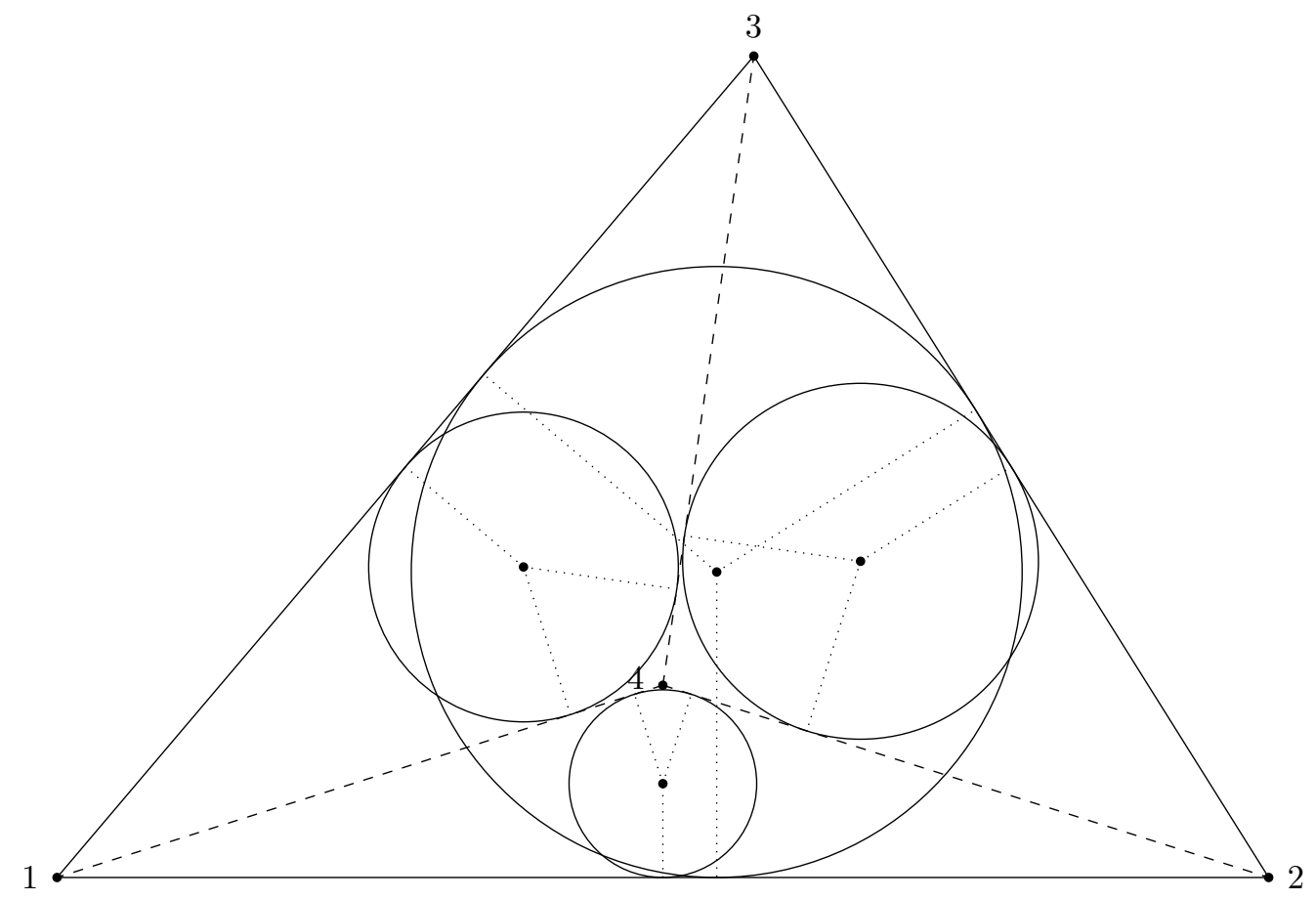




\section{Appendix A - New proof of the Eastwood-Norbury formula}

The four points in the Euclidean space $E^{3}$ viewed as $C \times R$ :

$P_{i}: x_{i}=\left(z_{i}, r_{i}\right), z_{i} \in \mathbb{C}, r_{i} \in \mathbb{R}$

$R_{i j}:=r_{i j}+r_{i}-r_{j}, z_{i j}:=z_{i}-z_{j}$

$R_{i j} R_{j i}=r_{i j}^{2}-\left(r_{i}-r_{j}\right)^{2}=\left|z_{i j}\right|^{2}=-z_{i j} z_{j i}$

$p_{1}=\left(z+\frac{\overline{z_{12}}}{R_{12}}\right)\left(z+\frac{\overline{z_{13}}}{R_{13}}\right)\left(z+\frac{\overline{z_{14}}}{R_{14}}\right)$

$p_{2}=\left(z+\frac{\overline{z_{21}}}{R_{21}}\right)\left(z+\frac{\overline{z_{23}}}{R_{23}}\right)\left(z+\frac{\overline{z_{24}}}{R_{24}}\right)$

$p_{3}=\left(z+\frac{\overline{z_{31}}}{R_{31}}\right)\left(z+\frac{\overline{z_{32}}}{R_{32}}\right)\left(z+\frac{\overline{z_{34}}}{R_{34}}\right)$

$p_{4}=\left(z+\frac{\overline{z_{41}}}{R_{41}}\right)\left(z+\frac{\overline{z_{42}}}{R_{42}}\right)\left(z+\frac{\overline{z_{43}}}{R_{43}}\right)$

Matrix of coefficients of $\left\{p_{1}, p_{2}, p_{3}, p_{4}\right\}$

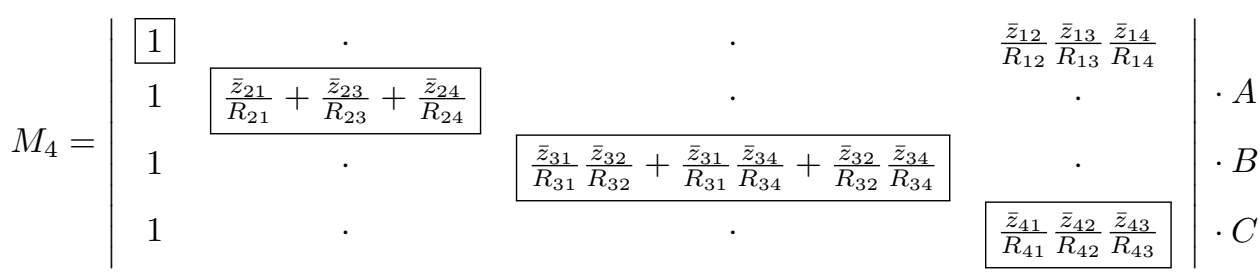

$A=z_{21}, B=z_{31} z_{32}, C=z_{41} z_{42} z_{43}$

Normalized Atiyah determinant

$$
\begin{aligned}
& D_{4}=\underbrace{\operatorname{det}\left(M_{4}\right)}_{\text {antisym. }} \cdot \underbrace{z_{21} \cdot z_{31} z_{32} \cdot z_{41} z_{42} z_{43}}_{\text {antisym. }}=\sum 1 \cdot\left(\frac{z_{21} \bar{z}_{21}}{R_{21}}+\frac{z_{21} \bar{z}_{23}}{R_{23}}+\frac{z_{21} \bar{z}_{24}}{R_{24}}\right) . \\
& \cdot\left(\frac{z_{31} \bar{z}_{31} z_{32} \bar{z}_{32}}{R_{31} R_{32}}+\frac{z_{31} \bar{z}_{31} z_{32} \bar{z}_{34}}{R_{31} R_{34}}+\frac{z_{32} \bar{z}_{32} z_{32} \bar{z}_{34}}{R_{32} R_{34}}\right) R_{14} R_{24} R_{34}= \\
& =\sum(R_{12} R_{24}+\underbrace{z_{21} z_{24}})(R_{13} R_{23} R_{34}+R_{13} \underbrace{z_{32} \bar{z}_{34}}+R_{23} \underbrace{z_{34}}_{R_{31} \bar{z}_{34}}) R_{14}+ \\
& +(R_{13} R_{24} R_{34} \underbrace{z_{21} \bar{z}_{23}}+R_{13} R_{24} R_{32} \underbrace{z_{21} \bar{z}_{34}}+R_{24} \underbrace{z_{21} \bar{z}_{23} z_{31} \bar{z}_{34}}) R_{14}
\end{aligned}
$$

(where summations are over all permutations of indices).

By writing $z_{i j} \bar{z}_{k l}=C[i, j, k, l]+\sqrt{-1} S[i, j, k, l]$ and using a Lagrange identity (involving the dot product of two cross products; a fact mentioned by N. Wildberger to the author) we have $S[i, j, k, l] S[p, q, r, s]=C[i, j, p, q] C[k, l, r, s]-C[i, j, r, s] C[k, l, p, q]$ (we have discovered this identity independently) and using the formula

$$
\begin{aligned}
C[i, j, k, l]=\operatorname{Re}\left(z_{i j} \bar{z}_{k l}\right) & =\frac{1}{2}\left[\left|z_{i l}\right|^{2}+\left|z_{j k}\right|^{2}-\left|z_{i k}\right|^{2}-\left|z_{j l}\right|^{2}\right]= \\
& =\frac{1}{2}\left[r_{i l}^{2}+r_{j k}^{2}-r_{i k}^{2}-r_{j l}^{2}\right]-\left(r_{i}-r_{j}\right)\left(r_{k}-r_{l}\right)
\end{aligned}
$$

we obtain our derivation of the Eastwood-Norbury formula. 
By this new method we obtained a polynomial formula for the planar configurations of 5 points (by $S_{5}$-symmetrization of a "one page" expression) and a rational formula for the spatial 5 point configuration (this last formula has almost 100000 terms).

This settles one of the Eastwood-Norbury conjectures. We do not yet have definite geometric interpretations for the "nonplanar" part of the formula involving heights $r_{i}$, $i=1, \ldots, 5$.

Our trigonometric (euclidean) Eastwood-Norbury formula (where $c_{i_{-} j k}:=\cos (i j, i k)$ and $\left.c_{i j_{-} k l}:=\cos (i j, k l)\right)$ :

$$
\begin{aligned}
16 R e\left(D_{4}\right)= & \left(1+c_{3 \_12}+c_{2 \_34}\right)\left(1+c_{1 \_24}+c_{4 \_13}\right)+ \\
& \left(1+c_{2 \_13}+c_{3 \_24}\right)\left(1+c_{4 \_12}+c_{1 \_34}\right)+ \\
& \left(1+c_{3 \_12}+c_{1 \_34}\right)\left(1+c_{2 \_14}+c_{4 \_23}\right)+ \\
& \left(1+c_{1 \_23}+c_{3 \_14}\right)\left(1+c_{2 \_34}+c_{4 \_12}\right)+ \\
& \left(1+c_{2 \_13}+c_{1 \_24}\right)\left(1+c_{3 \_14}+c_{4 \_23}\right)+ \\
& \left(1+c_{1 \_23}+c_{2 \_14}\right)\left(1+c_{3 \_24}+c_{4 \_13}\right)+ \\
& 2\left(c_{14 \_23} c_{13 \_24}-c_{14 \_23} c_{12 \_34}+c_{13 \_24} c_{12 \_34}\right)+ \\
& 72(\text { normalized volume })^{2} .
\end{aligned}
$$

Open problems: Hyperbolic (euclidean) version for $n \geq 4(n \geq 5)$ points in terms of distances, or in terms of angles. 


\section{Appendix B}

Here we first recall a remarkable inequality of I. Schur (c.f. J. Michael Steele: The Cauchy-Schwarz Master Class, Cambridge University Press, 2004.)

For all values $x, y, z \geq 0$ and all $\alpha \geq 0$ we have

$$
\begin{aligned}
I_{\alpha}(x, y, z): & =\sum x^{\alpha}(x-y)(x-z)= \\
& =x^{\alpha}(x-y)(x-z)+y^{\alpha}(y-x)(y-z)+z^{\alpha}(z-x)(z-y) \geq 0
\end{aligned}
$$

with equality iff either $x=y=z$ or two of the variables are equal and the third is zero. Note that $I_{\alpha}$ is a symmetric function. For a proof we can assume $0 \leq x \leq y \leq z$. Then clearly $x^{\alpha}(x-y)(x-z) \geq 0$ and by grouping the other two terms we get $(z-y)\left[z^{\alpha}(z-x)-y^{\alpha}(y-x)\right] \geq 0$ by observing that $z \geq y$ and $z-x \geq y-x$.

Now we state and prove several properties of a function

$$
d_{3}(x, y, z):=(x+y-z)(x-y+z)(-x+y+z) \quad(x, y, z \geq 0)
$$

which frequently appears in the main part of this paper.

We note that the area $A=A(a, b, c)$ of a triangle with sides lengths $a, b, c$ is given, according to the Heron-s formula:

$$
\begin{aligned}
(4 A)^{2} & =(a+b+c)(a+b-c)(a-b+c)(-a+b+c) \\
& =(a+b+c) d_{3}(a, b, c) \\
& =2 a^{2} b^{2}+2 a^{2} c^{2}+2 b^{2} c^{2}-a^{4}-b^{4}-c^{4}
\end{aligned}
$$

Properties of the function $d_{3}$ :

Proposition 2.13 We have the following identities and inequalities:

1. $x y z-d_{3}(x, y, z)=\sum x(x-y)(x-z) \geq 0$

2. $\left(x^{2}+y^{2}+z^{2}\right) d_{3}(x, y, z) \leq x y z(x y+x z+y z)$

3. $d_{3}(x, y, z)^{2}-d_{3}\left(x^{2}, y^{2}, z^{2}\right)=$

$=\sum x^{2}\left(y^{2}-y z+z^{2}-x^{2}\right)^{2}+\left(\sum x\left(y^{2}-y z+z^{2}-x^{2}\right)\right)^{2} \geq 0$

4. $d_{3}(x, y, z)^{2}-d_{3}\left(x^{2}, y^{2}, z^{2}\right)=$

$=8 x^{2} y^{2} z^{2}-2\left(x y z+x^{3}+y^{3}+z^{3}\right) d_{3}(x, y, z) \geq 0$

5. $(x+y+z)^{2} d_{3}(x, y, z)^{2}-3\left(x^{2}+y^{2}+z^{2}\right) d_{3}\left(x^{2}, y^{2}, z^{2}\right)=$ $=4 \sum x^{4}\left(x^{2}-y^{2}\right)\left(x^{2}-z^{2}\right) \geq 0$

6. $(x+y+z)(X+Y+Z) d_{3}(x, y, z) d_{3}(X, Y, Z)-3(x X+y Y+z Z) d_{3}(x X, y Y, z Z)=$ $2 \sum\left(x^{2}\left(x^{2}-y^{2}\right) X^{2}\left(X^{2}-Z^{2}\right)+X^{2}\left(X^{2}-Y^{2}\right) x^{2}\left(x^{2}-z^{2}\right)\right)+\left(x^{2}\left(Y^{2}-Z^{2}\right)+\right.$ $\left.y^{2}\left(Z^{2}-X^{2}\right)+z^{2}\left(X^{2}-Y^{2}\right)\right)^{2} \geq 0$ 


\section{Proof .}

All identities 1.-6. can be easily checked by expansion. The inequality in 1. follows from Schur's inequality $(\alpha=1)$, in 3. it is evident since the rhs is the sum of four squares (see [5]). Case 4. follows from 3. Case 5. follows from Schur's inequality $(\alpha=2)$. Case 6 . follows from a generalization of the case $\alpha=2$ of Schur's inequality:

$$
\begin{aligned}
& I I_{2}(x, y, z, X, Y, Z)=\sum x(x-y) X(X-Z)= \\
& =x(x-y) X(X-Z)+y(y-x) Y(Y-Z)+z(z-x) Z(Z-Y) \geq 0
\end{aligned}
$$

(by letting $y=x+h, z=y+k, Y=X+H, Z=Y+K, h, k, H, K \geq 0$ ).

Corollary 2.14 From the Proposition we get the following inequalities:

$$
d_{3}(x, y, z) \leq x y z \quad(\text { from } 1 .)
$$

and a stronger inequality $d_{3}(x, y, z) \leq 4 x^{2} y^{2} z^{2} /\left(x y z+x^{3}+y^{3}+z^{3}\right)$ (from 4.)

From 3. we have the inequality

$$
d_{3}(x, y, z)^{2} \geq d_{3}\left(x^{2}, y^{2}, z^{2}\right)
$$

which can also be obtained from 5. (which implies famous Finsler-Hadwiger inequality) by using the inequality $(x+y+z)^{2} \leq 3\left(x^{2}+y^{2}+z^{2}\right)$.

The inequality 6 . , with the help of Chebyshev inequality

$$
(x+y+z)(X+Y+Z) \leq 3(x X+y Y+z Z) \quad(x \leq y \leq z, \quad X \leq Y \leq Z)
$$

gives us the following inequality (which seems to be new):

$$
d_{3}(x, y, z) d_{3}(X, Y, Z) \geq d_{3}(x X, y Y, z Z)
$$

(when $0 \leq x \leq y \leq z, 0 \leq X \leq Y \leq Z$ ).

Remark 2.15 If $a, b, c$ are side lengths of a triangle then inequality $d_{3}(a, b, c) \leq$ abc follows also directly from the following identity

$$
a b c-d_{3}(a, b, c)=\frac{1}{2}\left[(-a+b+c)(b-c)^{2}+(a-b+c)(a-c)^{2}+(a+b-c)(a-b)^{2}\right]
$$

from which we also have the following inequality

$$
8\left(a b c-d_{3}(a, b, c)\right)^{3} \geq 27 d_{3}(a, b, c)(a-b)^{2}(a-c)^{2}(b-c)^{2}
$$




\section{Appendix C - Equations for mixed Atiyah 3pt energies}

$$
\begin{aligned}
& \Delta_{3} \quad=D_{3}-1=e^{-p} \frac{\sinh (p-a) \sinh (p-b) \sinh (p-c)}{\sinh (a) \sinh (b) \sinh (c)}, \quad\left(p=\frac{a+b+c}{2}\right) \\
& \Delta_{3}^{+} \quad=D_{3}^{+}-1=e^{p} \frac{\sinh (p-a) \sinh (p-b) \sinh (p-c)}{\sinh (a) \sinh (b) \sinh (c)} \\
& P_{1} \quad=\left(X-\Delta_{3}\right)\left(X-\Delta_{3}^{+}\right)= \\
& =X^{2}-2 \frac{\cosh (p) \sinh (p-a) \sinh (p-b) \sinh (p-c)}{\sinh (a) \sinh (b) \sinh (c)} X+\frac{\sinh ^{2}(p-a) \sinh ^{2}(p-b) \sinh ^{2}(p-c)}{\sinh ^{2}(a) \sinh ^{2}(b) \sinh ^{2}(c)} \\
& \Delta_{3}^{(1)} \quad=D_{3}^{001}-1=-e^{-(p-c)} \frac{\sinh (p) \sinh (p-a) \sinh (p-b)}{\sinh (a) \sinh (b) \sinh (c)}=-e^{-p+c} \frac{\sinh (p)}{\sinh (c)} \sin ^{2}(A) \\
& \Delta_{3}^{(6)}=D_{3}^{110}-1=-e^{p-c} \frac{\sinh (p) \sinh (p-a) \sinh (p-b)}{\sinh (a) \sinh (b) \sinh (c)}=-e^{+p-c} \frac{\sinh (p)}{\sinh (c)} \sin ^{2}(A) \\
& P_{2} \quad=\left(X-\Delta_{3}^{(1)}\right)\left(X-\Delta_{3}^{(6)}\right)= \\
& =X^{2}-2 \frac{\sinh (p) \sinh (p-a) \sinh (p-b) \cosh (p-c)}{\sinh (a) \sinh (b) \sinh (c)} X+\frac{\sinh ^{2}(p) \sinh ^{2}(p-a) \sinh ^{2}(p-b)}{\sinh ^{2}(a) \sinh ^{2}(b) \sinh ^{2}(c)} \\
& P_{3} \quad=\left(X-\Delta_{3}^{(2)}\right)\left(X-\Delta_{3}^{(5)}\right)= \\
& =X^{2}-2 \frac{\sinh (p) \sinh (p-a) \cosh (p-b) \sinh (p-c)}{\sinh (a) \sinh (b) \sinh (c)} X+\frac{\sinh ^{2}(p) \sinh ^{2}(p-a) \sinh ^{2}(p-c)}{\sinh ^{2}(a) \sinh ^{2}(b) \sinh ^{2}(c)} \\
& P_{4} \quad=\left(X-\Delta_{3}^{(3)}\right)\left(X-\Delta_{3}^{(4)}\right)= \\
& =X^{2}-2 \frac{\sinh (p) \cosh (p-a) \sinh (p-b) \sinh (p-c)}{\sinh (a) \sinh (b) \sinh (c)} X+\frac{\sinh ^{2}(p) \sinh ^{2}(p-b) \sinh ^{2}(p-c)}{\sinh ^{2}(a) \sinh ^{2}(b) \sinh ^{2}(c)} \\
& P(X)=P_{1} P_{2} P_{3} P_{4}=\prod_{i=0}^{7}\left(X-\Delta^{(i)}\right)=X^{8}+\cdots+e_{7} X-e_{8} \\
& e_{8} \quad=\prod_{i=0}^{7} \Delta^{(i)}=\frac{\sinh ^{6}(p) \sinh ^{6}(p-a) \sinh ^{6}(p-b) \sinh ^{6}(p-c)}{\sinh ^{6}(a) \sinh ^{6}(b) \sinh ^{6}(c)} \\
& e_{8} \quad=\frac{\left(1-c_{1}^{2}\right)^{2}\left(1-c_{2}^{2}\right)^{2}\left(1-c_{3}^{2}\right)^{2}}{4096}=\frac{\sin ^{4}(A) \sin ^{4}(B) \sin ^{4}(C)}{4096} \\
& e_{7} \quad=\frac{1}{256}\left(1-c_{1}^{2}\right)\left(1-c_{2}^{2}\right)\left(1-c_{3}^{2}\right)\left(-1+2 c_{1} c_{2} c_{3}+c_{1}^{2}+c_{2}^{2}+c_{3}^{2}\right)=\frac{1}{256} \sin ^{2}(A) \sin ^{2}(B) \sin ^{2}(C) \Phi^{2} \\
& P(X)=X^{8}+2 X^{7}+\frac{1}{4}\left(4+\sigma_{1}-\Phi^{2}\right) X^{6}+\frac{1}{4}\left(\sigma_{1}-2 \Phi^{2}\right) X^{5}-\frac{1}{32}\left(3 \sigma_{3}-2 \sigma_{2}+\left(2 \sigma_{1}-8\right) \Phi^{2}\right) X^{4}- \\
& -\frac{1}{32}\left(\sigma_{3}+2 \sigma_{1} \Phi^{2}\right) X^{3}-\frac{1}{256}\left(\sigma_{1} \sigma_{3}+\left(4 \sigma_{2}-\sigma_{3}\right) \Phi^{2}\right) X^{2}-\frac{1}{256} \sigma_{3} \Phi^{2} X+\frac{1}{4096} \sigma_{3}^{2} \\
& \text { where } \sigma_{1}=\sin ^{2}(A)+\sin ^{2}(B)+\sin ^{2}(C), \sigma_{2}=\sin ^{2}(A) \sin ^{2}(B)+\sin ^{2}(A) \sin ^{2}(C)+\sin ^{2}(B) \sin ^{2}(C) \text {, } \\
& \sigma_{3}=\sin ^{2}(A) \sin ^{2}(B) \sin ^{2}(C), \Phi^{2}=4 \cos (\sigma) \cos (\sigma-A) \cos (\sigma-B) \cos (\sigma-C), \sigma=\frac{A+B+C}{2} \\
& \prod_{i=0}^{7} D_{3}^{(i)}=\frac{1}{128}\left(\sigma_{3}-2 \sigma_{2}\right) \Phi^{2}+\frac{1}{4096}\left(\sigma_{3}^{2}-16 \sigma_{1} \sigma_{3}+256\left(\sigma_{2}-\sigma_{3}\right)\right)
\end{aligned}
$$




$$
\begin{aligned}
& P_{-}(X) \quad=X^{4}+(1+\Phi) X^{3}+\frac{1}{4}\left(c_{1}^{2}+c_{2}^{2}+c_{3}^{2}+3 c_{1} c_{2} c_{3}+3 \Phi\right) X^{2}+\frac{1}{8}\left(-1+2 c_{1} c_{2} c_{3}+c_{1}^{2}+c_{2}^{2}+c_{3}^{2}+\right. \\
& \left.+\left(1+c_{1} c_{2} c_{3}\right) \Phi\right) X-\frac{1}{64}\left(1-c_{1}^{2}\right)\left(1-c_{2}^{2}\right)\left(1-c_{3}^{2}\right) \\
& =\frac{1}{8} X(2 X+\Phi)\left[(2 X+1)(2 X+1+\Phi)+c_{1} c_{2} c_{3}\right]-\frac{1}{64}\left(1-c_{1}^{2}\right)\left(1-c_{2}^{2}\right)\left(1-c_{3}^{2}\right) \\
& =\frac{1}{8} X(2 X+\Phi)\left[(2 X+1)(2 X+1+\Phi)+\frac{1+\Phi^{2}-\left(c_{1}^{2}+c_{2}^{2}+c_{3}^{2}\right)}{2}\right]-\frac{1}{64}\left(1-c_{1}^{2}\right)\left(1-c_{2}^{2}\right)\left(1-c_{3}^{2}\right) \\
& =\frac{1}{16} X(2 X+\Phi)\left[2(2 X+1)(2 X+1+\Phi)-2+s_{1}^{2}+s_{2}^{2}+s_{3}^{2}\right]-\frac{1}{64} s_{1}^{2} s_{2}^{2} s_{3}^{2} \\
& P(X) \quad=P_{-}(X) P_{+}(X), \text { where } P_{+}(X)=\left.P_{-}(X)\right|_{\Phi \rightarrow-\Phi} \\
& \left.P_{-}(X)\right|_{\Phi=0}=\frac{1}{16} X \cdot 2 X\left[2(2 X+1)^{2}-2+s_{1}^{2}+s_{2}^{2}+s_{3}^{2}\right]-\frac{1}{64} s_{1}^{2} s_{2}^{2} s_{3}^{2} \\
& =X^{3}(X+1)+\frac{1}{8}\left(s_{1}^{2}+s_{2}^{2}+s_{3}^{2}\right) X^{2}-\frac{1}{64} s_{1}^{2} s_{2}^{2} s_{3}^{2} \\
& P(0) \quad=\frac{1}{4096} s_{1}^{4} s_{2}^{4} s_{3}^{4} \\
& \prod_{i=0}^{7} D_{3}^{(i)} \quad=P(-1)=\left[\frac{1}{16}(-1)(-2+\Phi)\left[-2(-1+\Phi)-2+s_{1}^{2}+s_{2}^{2}+s_{3}^{2}\right]-\frac{1}{64} s_{1}^{2} s_{2}^{2} s_{3}^{2}\right] \text {. } \\
& \cdot\left[\frac{1}{16}(-1)(-2-\Phi)\left[-2(-1-\Phi)-2+s_{1}^{2}+s_{2}^{2}+s_{3}^{2}\right]-\frac{1}{64} s_{1}^{2} s_{2}^{2} s_{3}^{2}\right] \\
& =\left[\frac{1}{16}(2-\Phi)\left[-2 \Phi+\sigma_{1}\right]-\frac{1}{64} \sigma_{3}\right]\left[\frac{1}{16}(2+\Phi)\left[2 \Phi+\sigma_{1}\right]-\frac{1}{64} \sigma_{3}\right] \\
& =\left[\frac{1}{16}\left(2 \Phi^{2}-4 \Phi+2 \sigma_{1}-\Phi \sigma_{1}\right)-\frac{1}{16} \sigma_{3}\right]\left[\frac{1}{16}\left(2 \Phi^{2}+4 \Phi+2 \sigma_{1}+\Phi \sigma_{1}\right)-\frac{1}{16} \sigma_{3}\right] \\
& =\left[\frac{1}{16}\left(2 \Phi^{2}+2 \sigma_{1}\right)-\frac{1}{64} \sigma_{3}-\frac{1}{16}\left(4+\sigma_{1}\right) \Phi\right]\left[\frac{1}{16}\left(2 \Phi^{2}+2 \sigma_{1}\right)-\frac{1}{64} \sigma_{3}+\frac{1}{16}\left(4+\sigma_{1}\right) \Phi\right] \\
& =\frac{1}{64^{2}}\left\{\left[4\left(\Phi^{2}+\sigma_{1}\right)-\sigma_{3}\right]^{2}-4^{2}\left(4+\sigma_{1}\right)^{2} \Phi^{2}\right\} \text {. }
\end{aligned}
$$


Svrtan, Urbiha Atiyah-Sutcliffe conjectures for four points configurations

\section{References}

[1] M. Atiyah. The geometry of classical particles. Surveys in Differential Geometry (International Press) 7 (2001).

[2] M. Atiyah. Configurations of points. Phil. Trans. R. Soc. Lond. A 359 (2001), 1375-1387.

[3] M. Atiyah and P. Sutcliffe. Polyhedra in Physics, Chemistry and Geometry, Milan Journal of Mathematics, Vol 71, Number 1/September 2003, 33-58

[4] M. Atiyah and P. Sutcliffe. The geometry of point particles. Royal Society of London Proceedings Series A, vol. 458, Issue 2021., 1089.-1116.

[5] C. E. Carroll, C. C. Yang and S. Ahn, Some triangle inequalities and generalizations, Canad. Math. Bull. Vol. 23(3), 1980, 267-274

[6] M. Eastwood and P. Norbury, A proof of Atiyah's conjecture on configurations of four points in Euclidean three-space. Geometry 83 Topology 5 (2001), 885-893.

[7] D.Ž. Đoković, Proof of Atiyah's conjecture for two special types of configurations, arXiv:math.GT/0205221 v4, 11 June 2002. Electron. J. Linear Algebra 9 (2002), 132-137.

[8] Đoković, D. Ž., Verification of Atiyah's conjecture for some nonplanar configurations with dihedral symmetry.In Publ. Inst. Math., Nouv. Sr. 72(86), (2002) 23-28.

[9] Mazen Bou Khusan, Michael Johnson On the conjecture regarding the fourpoint Atiyah determinant, SIGMA 10 (2014.), 070, 9 pages

[10] I. G. Macdonald Symmetric functions and Hall polynomials $2^{\text {nd }}$ edition, Oxford University Press, 1995.

[11] Joseph Malkoun, On the Atiyah problem on Hiperbolic Configurations of Four Points, arXive:150201364, 2015.; Geometrie Dedicata 2016., Vol 180, pp 287-292

[12] A. F. Möbius Über eine Methode, um von Relationen, welche der Longimetrie angehören, zu entsprechenden Sätzen der Planimetrie zu gelangen, Ber. Verhandl. königl. Sächs. Ges. Wiss. Leipzig Math. Phys. Cl., 1852., 41-45.

[13] D. Svrtan, Intrinsic Formula for Five Point (in euclidean plane) Atiyah Determinant, https://www.bib.irb.hr/553790 (2010)

[14] D. Svrtan, A proof of All three Euclidean Four Point Atiyah-Sutcliffe Conjectures, https://emis.de/journals/SLC/wpapers/s73vortrag/svrtan.pdf 
[15] D. Svrtan, A proof of All three Atiyah-Sutcliffe 4-point Conjectures, International Congress of Mathematicians, Seoul 2014, Abstracts Short Communication Poster Sessions, 136-137, S. Korea

[16] D. Svrtan, A progress on Atiyah-Sutcliffe geometric conjectures, MathChem-Comp, 2007., Dubrovnik, June 11.-16.

[17] D. Svrtan, I. Urbiha Atiyah-Sutcliffe Conjectures for Almost Collinear Configurations and Some New Conjectures for Symmetric Functions, math.AG/0406386

[18] D. Svrtan, I. Urbiha Verification and Strengthening of the Atiyah-Sutcliffe Conjectures for several types of Configurations, arXiv:math/0609174 (49 pages)

[19] J. Wolstenholme, A Book of Mathematical Problems on Subjects Included in the Cambridge Course, London and Cambridge, 1867, 56

[20] A.W.Walker, 'Problem 300', Nieuw Arch. Wisk. (3) 19 (1971), 224. 